\title{
A Systematic Approach for the Generation and Verification of Structural Hypotheses.
}

A short title:

\section{Generation and Verification of Structural Hypotheses}

Mikhail Elyashberg ${ }^{+}$, Kirill Blinov $^{+}$, Antony Williams ${ }^{\star}$.

+Advanced Chemistry Development, Moscow Department, 6 Akademik Bakulev Street, Moscow 117513, Russian Federation,

`ChemZoo Inc., 904 Tamaras Circle, Wake Forest, North Carolina 27587 


\begin{abstract}
During the process of molecular structure elucidation selection of the most probable structural hypothesis may be based on chemical shift prediction. The prediction is carried out using either empirical or quantum-mechanical (QM) methods. When QM methods are used NMR prediction commonly utilizes the GIAO option of the DFT approximation. In this approach the structural hypotheses are expected to be investigated by the scientist. In this article we hope to show that the most rational manner by which to create structural hypotheses is actually by the application of an expert system capable of deducing all potential structures consistent with the experimental spectral data and specifically using 2D NMR data. When an expert system is used the best structure(s) can be distinguished using chemical shift prediction is best performed either by an incremental or neural net algorithm. The time consuming quantum-mechanical calculations can then be applied, if necessary, to one or more of the "best" structures to confirm the suggested solution.
\end{abstract}

\title{
Keywords:
}

NMR $,{ }^{1} \mathrm{H},{ }^{13} \mathrm{C},{ }^{15} \mathrm{~N}$, expert system, GIAO, neural nets, increments.

\section{Introduction.}

The problem of molecular structure elucidation continues to be a major challenge and an important problem in the domain of organic chemistry and molecular spectroscopy. If a researcher is engaged in the investigation of a chemical reaction then one of his or her primary aims is to determine the structure of the reaction product(s).In the domain of natural products the isolation and elucidation of chemical structures can be a major challenge. In both cases the structure determination process, in general, is reduced to forming some structural hypotheses and then their subsequent verification. The generation of structural hypotheses is the initial step in the process of structure elucidation. 
Each hypothesis is the result of the comprehensive logical treatment of available spectral and chemical information associated with the structure under analysis. Nowadays the main source of spectral information is a combination of 1D and 2D NMR spectra complemented by the molecular formula and fragmentation ions determined from one of the many variants of mass spectrometry ${ }^{[1]}$. The information obtained from 2D NMR data is frequently rather complicated and fuzzy by nature. During the interpretation of 2D NMR data several structural hypotheses are often produced, each of them fitting the experimental data and commonly other available information. Hypothesis generation by humans offers a series of evident difficulties: 1) there is no guarantee that all possible hypotheses will be enumerated; 2) there are no criteria allowing the selection of the most credible hypotheses. As a result articles revising previously reported chemical structures can be quite common. A very interesting review reporting a great number of such cases was published recently by Nicolaou and Snyder ${ }^{[2]}$. The main objective of the article was to demonstrate to the chemical community that molecular structure elucidation is not a routine problem, and for the final decision it is frequently necessary not only to comprehensively utilize both spectral and X-ray data but also to confirm the structure via total synthesis. Different research teams offer different conclusions since investigators working in a traditional manner have no access to an exhaustive list of all possible candidate structures. We suggest that it is necessary to utilize a method that will systematically generate all possible structural hypotheses as well as identifying the most probable for this series. Choosing several of the most probable structures can drastically reduce the number of hypotheses which should be examined and finally verified and can both reduce the time consumed by highly qualified specialists as well as reduce the number of potential errors of misinterpretation.

Such methods already exist under the guise of Computer-Aided Structure Elucidation (CASE) techniques. A series of CASE expert systems have been described previously ${ }^{[3-6]}$. When these systems are used the relationships between spectral data and structural information are treated as a set of assumptions ("axioms") ${ }^{[7]}$. CASE programs derive all logical consequences 
(structures) from the set of axioms to solve the task which the spectroscopist performs when elucidating a structure without computational assistance. In other words, the initial assumptions and postulates form an axiomatic theory adjusted to solve a given analytical task. Any changes in the set of axioms (varying assumptions, adding new assumptions, etc.) influences the set of final structural hypotheses. Alternatively, if some new hypothetical structures are added by the researcher to an earlier set of candidate structures, it is necessary to verify that they are in agreement with the initial axioms. If a new candidate structure contradicts the initial axioms then either the new suggestion is erroneous or the given axioms should be revised. For example, a new candidate structure with a molecular formula or molecular mass differing from the experimentally determined values is only feasible when the corresponding MS data are in doubt.

An expert system can produce an output file containing many thousands and even tens of thousands of structures. All of them may formally meet the criteria and constraints provided by all of the 2D NMR correlations but the chemical shift assignments of the carbon and hydrogen nuclei for the majority of structures usually contradicts a number of the spectrum-structure correlations. A credible final structure or set of structures containing the correct structure can be distinguished from the full set of candidates using NMR spectrum prediction. The efforts of many groups of researchers have been focused on the development of algorithms for chemical shift prediction using different empirical approaches ${ }^{[8-12]}$.

Recently we developed two fast calculation algorithms ${ }^{[13-18]}$, one of which is based on additivity rules, the so-called incremental approach, and the other employs artificial neural networks. These algorithms provide a calculation speed of 3,000-10,000 ${ }^{13} \mathrm{C}$ chemical shifts per second with the average deviation between calculated and experimental chemical shifts equal to $d=1.8 \mathrm{ppm}$. The maximum calculation speed is achieved using the incremental approach. For a file containing tens of thousands of structural isomers the calculation time by either of the two methods is no longer than several minutes. Both algorithms are implemented in the CASE expert system Structure Elucidator ${ }^{[16-18]}$ and their high speed and accuracy has strongly influenced the 
CASE strategy ${ }^{[19]}$. The third algorithm included into the set of system predictors is based on a fragment method ${ }^{[1]}$. Although this method is not as fast as the other two, it allows the user to obtain a detailed explanation as to how each predicted chemical shift was calculated. These calculations use a HOSE-code (Hierarchical Organization of Spherical Environments) based prediction approach (see review ${ }^{[1]}$ ) and employ a database containing 175,000 structures with assigned ${ }^{13} \mathrm{C}$ and ${ }^{1} \mathrm{H}$ chemical shifts. For each atom within the candidate structure the related structures used for the prediction can be shown with their assigned chemical shifts and this allows the user to understand the origin of the predicted chemical shifts. All three methods can be used for ${ }^{1} \mathrm{H},{ }^{13} \mathrm{C},{ }^{15} \mathrm{~N},{ }^{19} \mathrm{~F}$ and ${ }^{31} \mathrm{P}$ NMR chemical shift prediction and all of them are implemented within the Structure Elucidator software program.

During the last decade there has been a significant growth in the number of publications devoted to the application of quantum-mechanical (QM) chemical shift calculations for identifying the most credible structure(s). It has been shown ${ }^{[20-25]}$ that a $\mathrm{QM}$ approach provides a calculation accuracy that is, in general, enough for the successful validation of candidate structures and, in particular, for the revision of structures which were originally determined incorrectly. One of the most recent and striking examples of the successful application of the QM approach was described by Rychnovsky ${ }^{[26]}$ who refuted the incorrect structure of hexacyclinol proposed by Grafe et $a^{[27]}$ and suggested another structure which was then unambiguously proved by synthesis and X-ray analysis ${ }^{[28]}$. A systematic method to confirm the structure of hexacyclinol using Structure Elucidator was also described elsewhere ${ }^{[29]}$. QM chemical shift prediction is rather time consuming relative to other approaches. Different authors report different time costs for such calculations depending on the molecules size, flexibility and the number of possible conformers for which chemical shifts should be predicted. The processor time can vary from several hours to several tens of hours. It is therefore necessary to reduce the number of candidate structures to which QM calculations should be applied as much as possible before starting a series of calculations. It is natural to expect that prior to performing QM 
chemical shift calculations a minimum set of candidate structures should be chosen on the basis of fast chemical shift prediction by empirical methods. Structure generation and subsequent ranking of the candidate structures in descending order of their probability consumes only a few minutes on a modern processor using today's expert systems ${ }^{[18]}$. Obviously the application of QM methods can play a decisive role in such cases when the analyzed structures contain "exotic" fragments that were absent from the training set. Another situation whereby QM prediction could help to identify a preferable structure may occur when the differences between the experimental NMR chemical shifts and those calculated by empirical methods for several "best structures" are too small to enable a certain choice.

In many publications the potential of QM methods was evaluated on molecules for which the number of heavy atoms was most frequently around 20 and rarely reached 30 atoms. Meanwhile, for example, many natural products molecules contain 40-100 or more heavy atoms and the prediction of chemical shifts for such molecules is not attainable by QM methods as yet. For large molecules we can only rely on empirical methods for chemical shift prediction.

It is worthy to note that some publications (for example ${ }^{[30]}$ ) devoted to structure elucidation assisted by QM chemical shift prediction frequently do not mention empirical methods at all. In many cases it is seemingly alleged that the QM approach is the unique predictive method for proving or disproving a proposed structure. Other works (for example ${ }^{[31,32]}$ ) compare the accuracy of QM methods with the accuracy of older versions of empirical approaches ${ }^{[33]}$ that do not appropriately represent the performance of contemporary programs ${ }^{[8,15,34-36]}$. To the best of our knowledge the capabilities of the newer empirical methods for identifying the most probable structure within a set of proposed structures have not been compared with results attained using QM methods until this publication.

With this in mind we selected a series of articles where the QM approach was successfully used for selection of the right structure among a series of suggested molecules or for revising the originally hypothesized chemical structure. For each case if 2D NMR data were available then 
we made an attempt to solve the problem systematically using the expert system Structure Elucidator $^{[16,17]}$ which is capable of enumerating all possible structural hypotheses. We found that the right structure was also assigned as the most probable one in the examples considered by both QM and fast empirical NMR chemical shift predictions ${ }^{[8,13]}$, while alternative and incorrect structures suggested by researchers were ranked lower. Securitization of the examples studied enabled us to suggest a general approach where the most probable structure is established as a result of the joint application of a CASE expert system in combination with both empirical and quantum-mechanical methods for chemical shift prediction.

\section{RESULTS AND DISCUSSION}

\section{Problems solved in Common Mode of expert system.}

There are two main modes for operating the Structure Elucidator (StrucEluc) expert system: the Common Mode and the Fragment Mode. In the Common Mode the program uses atoms included into the molecular formula and connectivities derived from 2D NMR spectra, with the atoms characterized by both their chemical shifts and their properties if available. The properties may include atom valence, hybridization, the possibility of having heteroatoms as neighbors, the number of hydrogen atoms attached to carbons adjacent to $\mathrm{CH}, \mathrm{CH}_{2}$ and $\mathrm{CH}_{3}$ groups, etc. The program infers all logical consequences (structures) resulting from the data outlined above. This mode of operation resembles, to a certain extent, an "ab initio" approach. In the Fragment Mode of the program "free" atoms and structural fragments are utilized as the basis of structure generation. The fragments can be found in a database or proposed by the chemist. This mode is especially effective in those cases where there is a lack of COSY or/and HMBC correlations.

In this section we will consider several examples in which the preferable structure should be selected on the basis of NMR spectrum prediction. These problems were used by different researchers to examine the possibilities of QM methods to be used as an analytical tool. We used spectral data presented in the corresponding publications to demonstrate how the problems could be solved using the StrucEluc system operating in the Common Mode. 


\section{Example 1. Boletunones $A$ and $B$}

Kim et $\mathrm{a}^{[37]}$ separated two new natural products and attributed them to Boletunone $\mathrm{A}(\mathbf{1})$ and Boletunone B (2) using 1D and 2D NMR data. Both structures are presented with the ${ }^{13} \mathrm{C}$ chemical shift assignment suggested by authors ${ }^{[37]}$ :

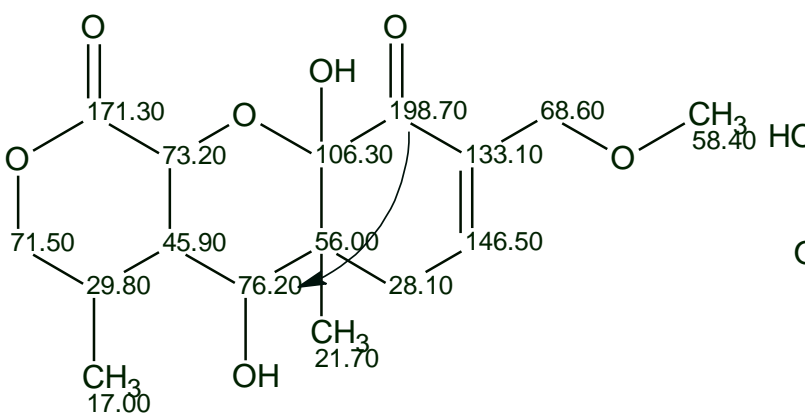

1

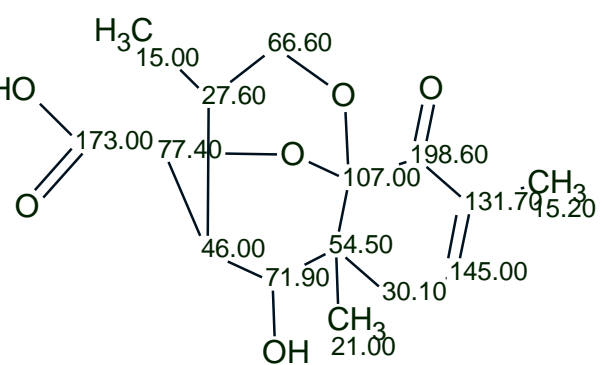

2

Steglich and Hellwig ${ }^{[38]}$ have shown that structures $\mathbf{1}$ and $\mathbf{2}$ are wrong, and the following alternative structural formulae for these compounds, $\mathbf{3}$ and $\mathbf{4}$, were offered and proved:

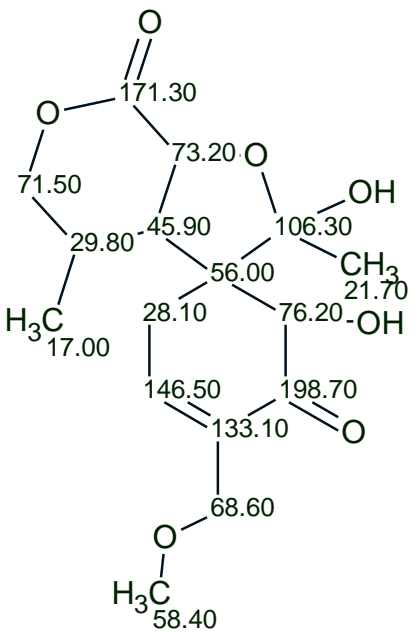

3

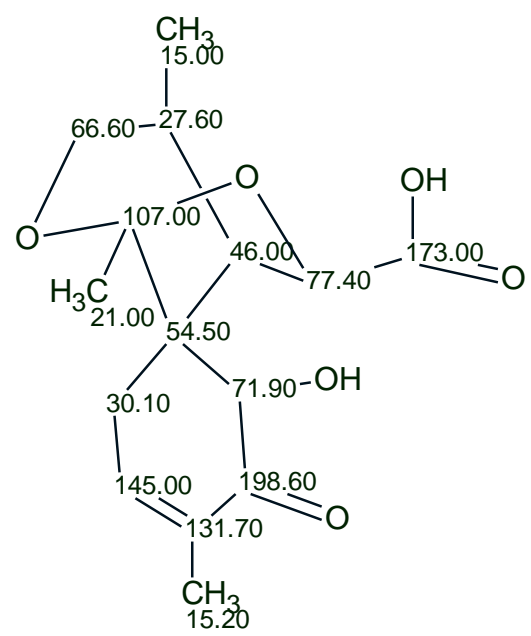

4

For Boletunone A, we have shown ${ }^{[19]}$ that application of StrucEluc allowed reliable determination of the right structure 3 in $0.15 \mathrm{~s}$. We also demonstrated how the problem of Boletunone B could be solved very quickly and correctly in a systematic way. Since the Boletunone B story was used by Bagno et $a^{[20]}$ for challenging DFT chemical shift calculations used as an analytical tool, we will explain here the solution of this problem in more detail. Bagno et al ${ }^{[20]}$ showed that QM based NMR chemical shift prediction was capable of distinguishing between the structural hypotheses of $\mathbf{2}$ and $\mathbf{4}$ and could determine the genuine 
structure. It was demonstrated that a quantum-chemical approach could be used to resolve disputes regarding the acceptance or rejection of different isomeric structures.

To calculate the ${ }^{1} \mathrm{H}$ and ${ }^{13} \mathrm{C}$ spectra for both the original proposal 2 and the revised structure 4 , the geometries were optimized at the B3LYP/6-31G(d,p) level in the gas phase, and the NMR properties were calculated with B3LYP/cc-pVTZ both in the gas phase and with a solvent reaction field of DMSO.

To make a choice as to the most favorable of the two structures, the following statistical characteristics were used: the parameters $a$ and $b$ for the linear regression equation $\delta_{\text {calcd }}=a+b \delta_{\text {exptl }} ;$ the correlation coefficient, $R^{2} ;$ the mean absolute error $(M A E)$ defined as $\Sigma_{n}\left|\delta_{\text {calcd }} \delta_{\text {exptl }}\right| / n$; the corrected mean absolute error, CMAE, defined as $\Sigma_{n}\left|\delta_{\text {corr }}-\delta_{\text {exptl }}\right| / n$, where $\delta_{\text {corr }}=\left(\delta_{\text {calcd }}-a\right) / b$ and therefore corrects for systematic errors. The corrected chemical shifts are referred to the scaled shifts.

The authors ${ }^{[20]}$ found that for the correct structure (4) $\operatorname{MAE}\left({ }^{13} \mathrm{C}\right)=7.2 \mathrm{ppm}, \operatorname{CMAE}\left({ }^{13} \mathrm{C}\right)=1.9 \mathrm{ppm}$ and $\mathrm{R}^{2}=0.9984$, while for the wrong structure, 2 , these values were equal to $6.0 \mathrm{ppm}, 3.7 \mathrm{ppm}$ and 0.9952 correspondingly. These parameters indicate that structure 4 is preferable. The calculation of the ${ }^{1} \mathrm{H}$ chemical shifts and coupling constants confirmed that the revised structure 4 is in better agreement with the experimental spectrum than the originally proposed structure 2 . An attempt to improve prediction accuracy by optimizing the structures at the MP2/cc-pVDZ level indicated that the changes in geometry were very small and NMR properties were very similar.

For systematic elucidation of the Boletunone B structure, we input the $1 \mathrm{D}$ and 2D NMR data associated with Boletunone B into the StrecEluc software. The ${ }^{1} \mathrm{H}$ signal multiplicities determined by Kim et $a l^{[37]}$ were introduced for all methyl groups and for two $\mathrm{CH}$ groups $(\delta \mathrm{C}=77.4, \delta \mathrm{H}=4.34(\mathrm{~d})$ and $\delta=71.9, \delta \mathrm{H}=3.77(\mathrm{~s}))$. The multiplicities of these groups had been determined with sufficient reliability. During the first program run "Strict Structure Generation" ${ }^{[17]}$ was performed. This meant that the absence of so-called "nonstandard" correlations ${ }^{[39]}$ in the 
2D NMR data was assumed, non-standard correlations defined as those for which the distance between intervening nuclei in the COSY and HMBC spectra is longer than 3 bonds.. The program generated 142 structures in $3.8 \mathrm{~s}, 57$ structures were retained after spectral filtering and no structures were removed after checking the structures for duplicates. The result can be symbolized in the following way: $k=142 \rightarrow 57 \rightarrow 57, t_{g}=3.8 \mathrm{~s}$.

Neither the $\mathbf{2}$ nor $\mathbf{4}$ structure were found among the 57 structures since the presence of one nonstandard correlation (NSC) in the HMBC NMR data was postulated both in the original and revised structures. ${ }^{13} \mathrm{C}$ chemical shift prediction was performed for all structures in the output file using the neural net $(\mathrm{NN})$ approach discussed previously and all structures were ranked in ascending order of the average chemical shift deviation $d_{N}\left({ }^{13} \mathrm{C}\right)$ calculated for each structure. A lower index $N$ denotes that the prediction was performed by the NN method. The top structures contained within the ranked structure file are presented in Figure 1.

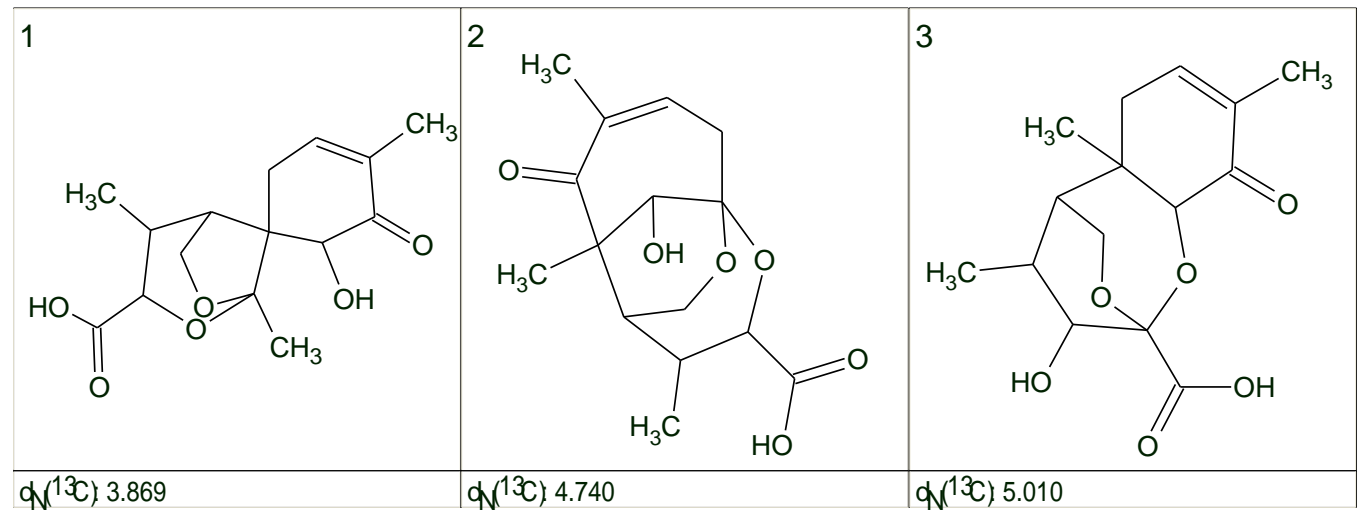

Figure 1. The three first structures of the file produced by Strict Structure Generation from the NMR data measured for Boletunone B. The structures are ranked in ascending order of average deviation $d_{N}\left({ }^{13} \mathrm{C}\right)$.

The average deviation value of the first ranked structure is twice as large as the average deviation of the NN method (1.8 ppm), which indicates a need to repeat structure generation in Fuzzy Mode $^{[18]}$. Structure generation was repeated in this mode allowing one NCS in the HMBC data as assumed by both groups of investigators ${ }^{[37,38]}$. This gave the following result:

$k=1211 \rightarrow 383 \rightarrow 374, t_{g}=14 \mathrm{~s}$ 
The top ranked structures in the file are shown in Figure 2.

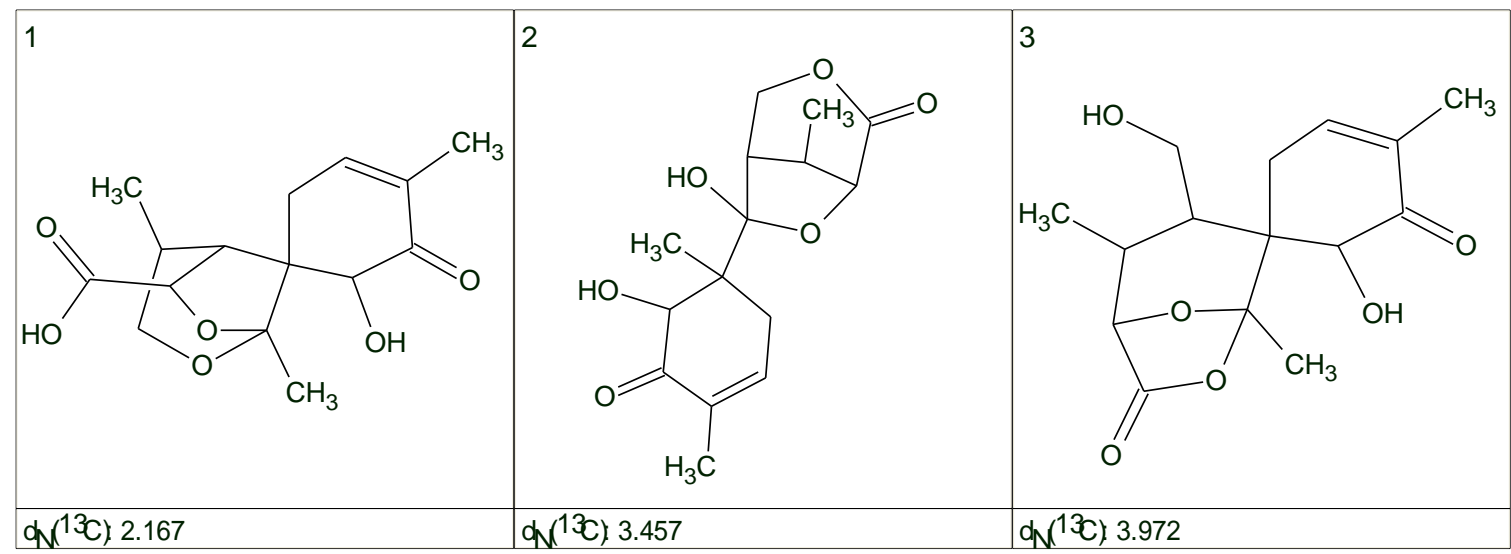

Figure 2. The top ranked structures in the output file resulted from Fuzzy Structure Generation using the NMR data obtained for Boletunone B. The top-ranked structure is identical to the revised structure 4.

Figure 2 shows that the revised structure is identical to the "best" structure and the difference between the average deviations calculated for the second and first structures is significant ${ }^{[18]}$. The original structure $\mathbf{2}$ was not found in the output file since structure $\mathbf{2}$ contradicts the multiplicity of the ${ }^{1} \mathrm{H}$ signal $\delta=3.77 \mathrm{ppm}$ : a singlet is observed in the ${ }^{1} \mathrm{H}$ spectrum, while a doublet should be expected if structure $\mathbf{2}$ was correct. Fuzzy Structure Generation was performed without taking into account the multiplicities $\left(\mathrm{k}=32814 \rightarrow 8621 \rightarrow 7533, t_{g}=1 \mathrm{~min} 10 \mathrm{~s}\right)$ produced structure 2 and it was ranked in third position in the resulting structure file $\left(d_{N}{ }^{13} \mathrm{C}\right.$ is equal to $3.08 \mathrm{ppm}$ for this structure). Direct comparison of the linear regression parameters calculated for structures $\mathbf{2}$ and $\mathbf{4}$ shows that $\mathrm{R}^{2}(\mathbf{4})=0.999$ and $\mathrm{R}^{2}(\mathbf{2})=0.995$ and these parameters are practically the same as those found by Bagno et al. The application of the systematic approach outlined here would allow researchers to immediately identify the correct structure that could then be further justified and investigated in more details by quantum-chemical calculations if it were deemed necessary. The regression lines associated with structures $\mathbf{2}$ and $\mathbf{4}$ are shown in Figures $1 \mathrm{~S}$ and $2 \mathrm{~S}$ (see Supporting Information). The figures show the difference in point scattering corresponding to the original and revised structures.

\section{Example 2.}


As well as articles devoted to the evaluation of DFT chemical shift calculation as a potential tool for identification of a proposed structure there are also publications where this approach has been applied to solving real chemical problems. Recently Sanz et al ${ }^{[30]}$ employed ${ }^{13} \mathrm{C}$ and ${ }^{15} \mathrm{~N}$ chemical shift calculations by the GIAO approximation of the DFT method to choose between the structures forming two pairs of isomers $(\mathbf{5}$ or $\mathbf{6})$ and $(\mathbf{7}$ or $\mathbf{8})$ :<smiles>FC(F)(F)c1cc(-c2ccccc2)nc2c(-c3ccccc3)cnn12</smiles>

5

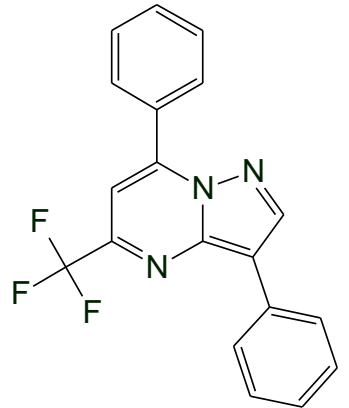

6

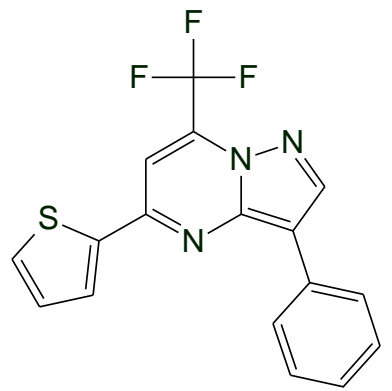

7

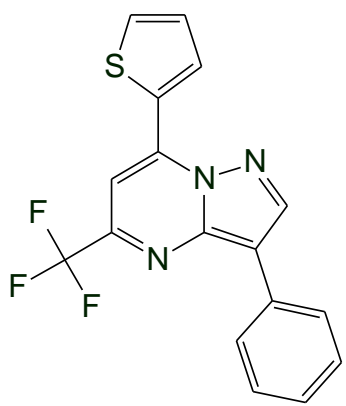

8

The authors ${ }^{[30]}$ synthesized and separated two samples, \#1 and \#2, with molecular formulae of $\mathrm{C}_{19} \mathrm{H}_{12} \mathrm{~F}_{3} \mathrm{~N}_{3}$ (1) and $\mathrm{C}_{17} \mathrm{H}_{10} \mathrm{~F}_{3} \mathrm{~N}_{3} \mathrm{~S}$ (2). For the purpose of structure determination and confirmation, 1D NMR spectra $\left({ }^{1} \mathrm{H},{ }^{13} \mathrm{C},{ }^{15} \mathrm{~N}\right.$ and $\left.{ }^{19} \mathrm{~F}\right)$ in combination with ${ }^{1} \mathrm{H}-{ }^{13} \mathrm{C}$ gs-HMBC and ${ }^{1} \mathrm{H}-{ }^{15} \mathrm{~N} g s-\mathrm{HMBC}$ data were used. The authors ${ }^{[30]}$ derived the two pairs of alternative isomers shown above and the problem was reduced to selection of the correct structure within each of the isomer pairs. For this purpose the authors calculated both the ${ }^{13} \mathrm{C}$ and ${ }^{15} \mathrm{~N}$ chemical shifts using the GIAO method of DFT approximation for two model compounds:<smiles>Cc1cc(C(F)(F)F)n2ncc(C)c2n1</smiles>

9 10

The model compounds $\mathbf{9}$ and $\mathbf{1 0}$ were used in order to simplify the QM computations. Linear regressions of the calculated versus experimental ${ }^{13} \mathrm{C}$ chemical shifts, the latter assigned for the proposed structures, allowed the authors ${ }^{[30]}$ to confirm configuration 9 as the most preferable $\left(\mathrm{R}^{2}(\mathbf{9})=0.998 ; \mathrm{R}^{2}(\mathbf{1 0})=0.984\right) .{ }^{15} \mathrm{~N}$ chemical shift prediction resulted in $\mathrm{R}^{2}(\mathbf{9})=0.992$ and 
$\mathrm{R}^{2}(\mathbf{1 0})=0.991$ values for the corresponding model structures and only slightly supports model 9 as the preferred structure. In order to show how the problem could be solved without utilizing model structures and QM calculations we repeated the structure elucidation process for both samples using our systematic approach.

The experimental 1D and 2D NMR data and the molecular formulae obtained for the two samples were input into the StrucEluc system and the two problems, 1 and 2, were solved. For the structure elucidation process both ${ }^{1} \mathrm{H}-{ }^{13} \mathrm{C}$ and ${ }^{1} \mathrm{H}-{ }^{15} \mathrm{~N}$ HMBC correlations were used as initial data.

Problem 1. Strict Structure Generation gave the following result: $k=106 \rightarrow 44 \rightarrow 20, t_{g}=27 \mathrm{~s}$. For all structures ${ }^{1} \mathrm{H},{ }^{13} \mathrm{C}$, and ${ }^{15} \mathrm{~N}$ chemical shifts were calculated using the $\mathrm{NN}$ approach and, following the general StrucEluc strategy, the resulting structure file was ranked in ascending order of $d_{N}\left({ }^{13} \mathrm{C}\right)$. The first three structures of the ranked file are presented in Figure 3.

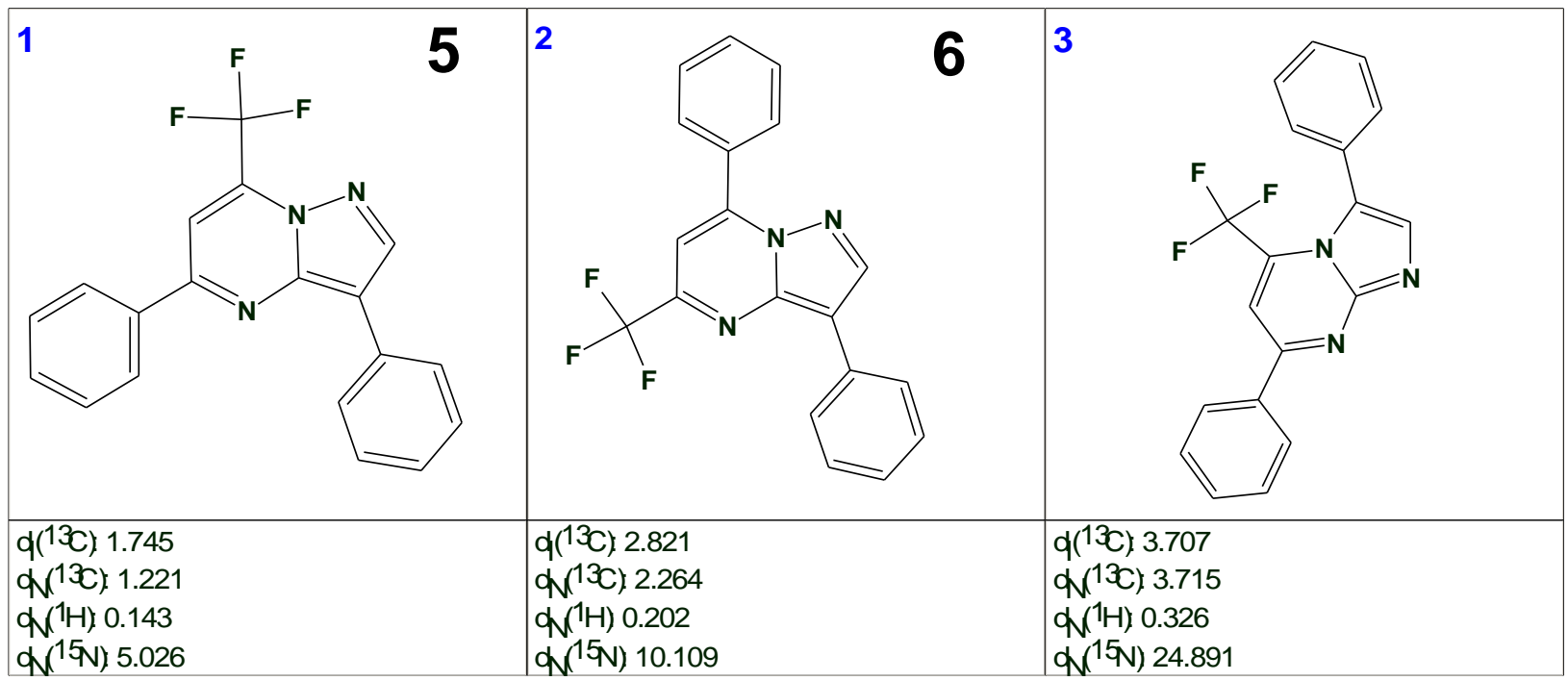

Figure 3. The first three structures of the ranked structural file produced as a solution for Problem 1. The right structure is confirmed by average deviations calculated for ${ }^{1} \mathrm{H},{ }^{13} \mathrm{C}$ and ${ }^{15} \mathrm{~N}$ spectra. $d_{I}\left({ }^{13} \mathrm{C}\right)$ denotes the average deviation found when the incremental method of chemical shift prediction was employed. 
The figure shows that all average deviations $-d_{N}\left({ }^{13} \mathrm{C}\right), d_{N}\left({ }^{1} \mathrm{H}\right), d_{N}\left({ }^{15} \mathrm{~N}\right)$ and $d_{I}\left({ }^{13} \mathrm{C}\right)$ - indicate that structure $\mathbf{5}$ is the most probable one. The values $R^{2}(\mathbf{5})=0.984$ and $R^{2}(\mathbf{6})=0.895$ calculated for ${ }^{13} C$ prediction by the NN method supports our assignment and the conclusion of Sanz et al ${ }^{[30]}$. Problem 2. The first program run was executed using the Strict Mode of structure generation. Only one structure, 11, was produced in $7 \mathrm{~s}$. The very large values of the calculated average deviations $\left(d_{N}\left({ }^{13} \mathrm{C}\right) \sim 9 \mathrm{ppm}, d_{N}\left({ }^{15} \mathrm{~N}\right) \sim 65 \mathrm{ppm}\right)$ suggested that structure 11 was likely incorrect and, consequently, nonstandard HMBC connectivities might exist within the 2D NMR data.

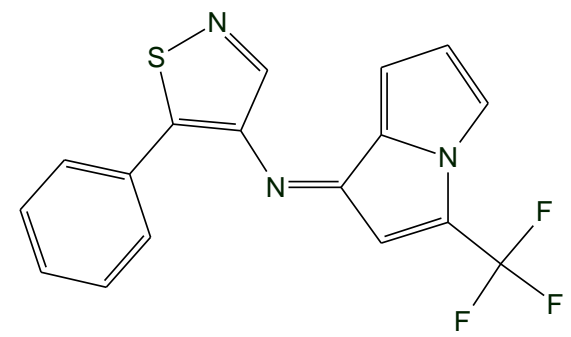

11

As mentioned earlier according to the general strategy of computer-aided structure elucidation developed by us ${ }^{[16-18,40]}$, in such a case Fuzzy Structure Generation ${ }^{[40]}$ should be carried out. Fuzzy Structure Generation was performed under the conditions $m=1-15, a=x$, that is the possible number of NSCs is allowed to be between 1 and 15, and lengthening of connectivities is replaced by their removal during structure generation. This mode allows problems to be solved when 2D NMR data contain an unknown number of NSCs having unknown lengths. The following result was obtained: $k=1481 \rightarrow 415 \rightarrow 164, t_{g}=11 \mathrm{~min}$.

Chemical shift prediction and structure ranking promoted structure 7 to the first ranked position while structure $\mathbf{8}$ was ranked as fourth. Both structures with average deviations are presented in Figure 4. 


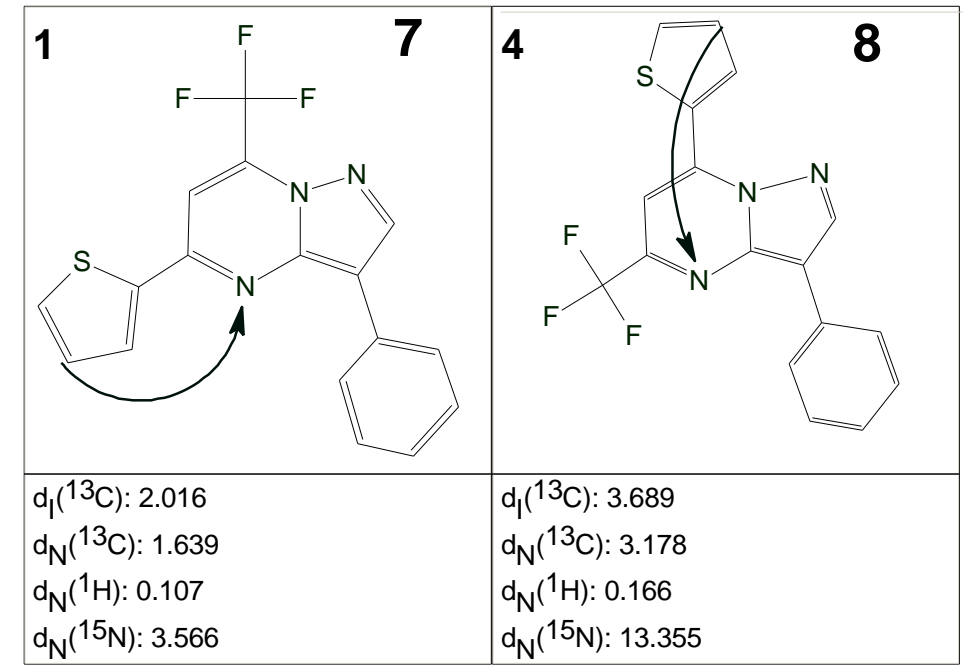

Figure 4. Structures 7 and 8 proposed in the work of Sanz ${ }^{[30]}$ with their calculated average deviations. The arrows show long-range ${ }^{1} \mathrm{H}_{-}{ }^{15} \mathrm{~N}$ HMBC correlations of ${ }^{5} \mathrm{~J}_{\mathrm{NH}}$ for structure 7 and ${ }^{7} J_{N H}$ for structure 8 . For these structures values of $R^{2}(7)=0.974$ and $R^{2}(8)=0.807$ were calculated.

In this case the preferable structure was also indicated by all NMR shift predictions having the lowest deviations as well as the calculated values of $\mathrm{R}^{2}(\mathbf{7})=0.974$ and $\mathrm{R}^{2}(\mathbf{8})=0.807$. Additional evidence for structure $\mathbf{7}$ is the need for a seven bond nonstandard correlation in structure $\mathbf{8}$.

In the examples discussed in this section empirical methods of chemical shift prediction are not only dramatically faster but, in definite cases, are even more reliable because no structural models simplifying the calculations were utilized. Nevertheless, the coincidence of structural assignments made by both QM and empirical approaches shows that researchers can choose the method that is more attractive for them. Graphs of linear regression and the associated scattering of points calculated for both problems can be found in the Supporting Information (Figures 3S-6S).

\section{Problems solved in Fragment Mode.}

The analytical process taken by experts for molecular structure elucidation from 2D NMR data has been described in a series of books (for example, ${ }^{[41-43]}$. The spectroscopist usually tries to assemble some fragments from atoms and their spectral signals and then commonly combine the fragments using HMBC correlations until a complete molecular structure or set of plausible 
structures are constructed. As previously discussed there is no guarantee that all possibilities will be taken into account by the process of manual structure assembly. Moreover, some superfluous structural hypotheses may be suggested. If selection of the right structure is performed by QM chemical shift prediction then additional time will be consumed for checking these structures. In this section we will demonstrate how an expert system supplied with new empirical chemical shift prediction methods can be used to assist the spectroscopist in obtaining the right solution in an optimal manner.

\section{Example 1.}

Balandina et ${ }^{[22]}$ synthesized a novel quinoxaline and determined its molecular formula $\mathrm{C}_{16} \mathrm{H}_{10} \mathrm{~N}_{2} \mathrm{O}_{2}$ from the MS data $(\mathrm{m} / \mathrm{z}=262(\mathrm{M}+)$ combined with elemental analysis data. To elucidate the structure of this compound, authors used ${ }^{1} \mathrm{H},{ }^{13} \mathrm{C}$ and ${ }^{15} \mathrm{~N}$ NMR spectra. Assignment of the ${ }^{1} \mathrm{H}$ and ${ }^{13} \mathrm{C}$ NMR spectra was accomplished using data derived from DEPT, 2D COSYGP, HSQC and HMBC experiments. Analysis of the NMR data provided two fragments containing $\mathrm{H}, \mathrm{C}$ and $\mathrm{N}$ atoms with assigned chemical shifts. Three quaternary carbons (151.04, 138.29 and 134.68) without HMBC correlations, one hydrogen atom and two oxygen atoms were not assigned to either of the fragments. The initial data for forming structural hypotheses is presented in Figure 5.
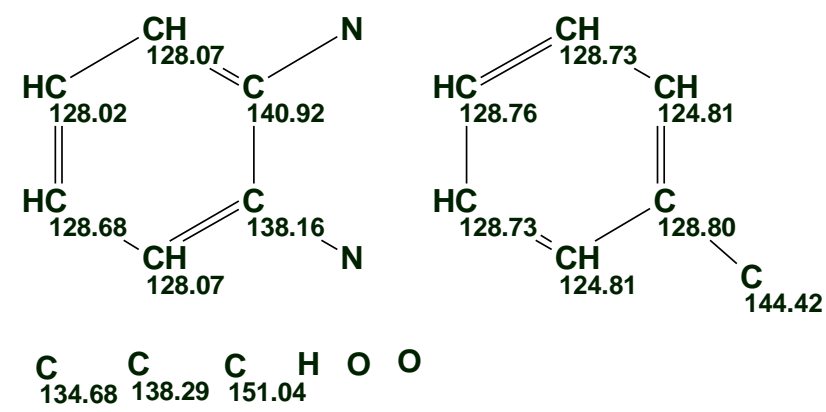

Figure 5. Initial structure information for the generation of structural hypotheses.

Using these data and some additional chemical considerations the authors suggested 6 structures which are presented in Figure 6. 
<smiles>O=C1Nc2ccccc2N2C1=C2C(=O)c1ccccc1</smiles>

12<smiles>O=c1oc2nc3ccccc3[nH]c-2c1-c1ccccc1</smiles>

13

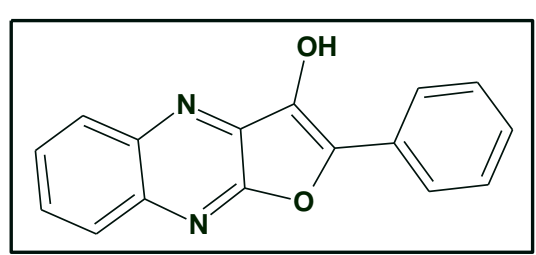

14<smiles>O=C1c2nc3ccccc3nc2OC1c1ccccc1</smiles>

15

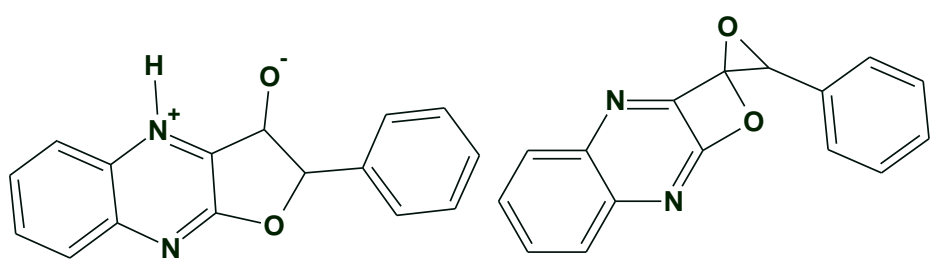

16
17

Figure 6. Six suggested structures derived from the experimental data. Structure $\mathbf{1 4}$ corresponds to the correct structure.

To select the right structure, ${ }^{1} \mathrm{H},{ }^{13} \mathrm{C}$ and ${ }^{15} \mathrm{~N}$ chemical shifts were predicted for structures $\mathbf{1 2}-\mathbf{1 7}$ using the DFT framework and using a hybrid exchange-correlation function, GIAO B3LYP, at the 6-31G(d) level. Full geometry optimizations were performed under ab initio RHF/6-31G conditions. Linear correlation coefficients of the experimental $v s$ calculated ${ }^{13} \mathrm{C}$ chemical shifts $\left(\mathrm{R}^{2}\right)$, root-mean-square errors (rms), slope (a), standard deviations (sd) and mean absolute deviations $(\mathrm{MAD}=\Sigma[\mid \delta \exp -\delta$ calc $\mid] / \mathrm{n})$ for structures 12-17 were computed. As a result structure 14 was identified as the most probable $\left(\mathrm{R}^{2}=0.9758, \mathrm{rms}=1.16 \mathrm{ppm}, \mathrm{sd}=1.2 \mathrm{ppm}, \mathrm{MAD}=7.03\right.$ $\mathrm{ppm})$. Other proposed structures were rejected by the authors due to smaller $\mathrm{R}^{2}$ values $\left(\mathrm{R}^{2}=0.01\right.$ $0.57)$ and larger deviations. It should be noted that the $\mathrm{R}^{2}$ values have a reasonable interpretation only in those cases when experimental chemical shifts are assigned to the atoms of competing structures. Otherwise, selection of the preferable structure can be attained only by simple comparison of the experimental with the calculated spectrum and based on determining outliers. Obviously the application of an expert system for structure elucidation provides 
chemical shift assignments that agree with the 2D NMR correlations and consequently the selection of the best structure occurs automatically.

To solve this problem using a CASE approach, spectral data presented in the work $^{[22]}$ were entered into the StrucEluc system. Fragments and atoms shown in Figure 5 were eventually transformed into a Molecular Connectivity Diagram $(\mathrm{MCD})^{[16]}$. The atom properties for three carbon atoms not included into the fragments were automatically set as sp2/not defined (atom hybridization is $s p^{2}$, possibility of neighboring heteroatoms is not defined). Structure generation was performed in the automatic mode and Fuzzy Structure Generation was allowed. The following result was obtained: $k=247 \rightarrow 16 \rightarrow 4, t_{g}=1 \mathrm{~s}$.

Empirical chemical shift prediction was performed for all nuclei. Subsequent structural ranking by $d_{N}\left({ }^{13} \mathrm{C}\right)$ deviation resulted in the structure ordering shown in Figure 7:

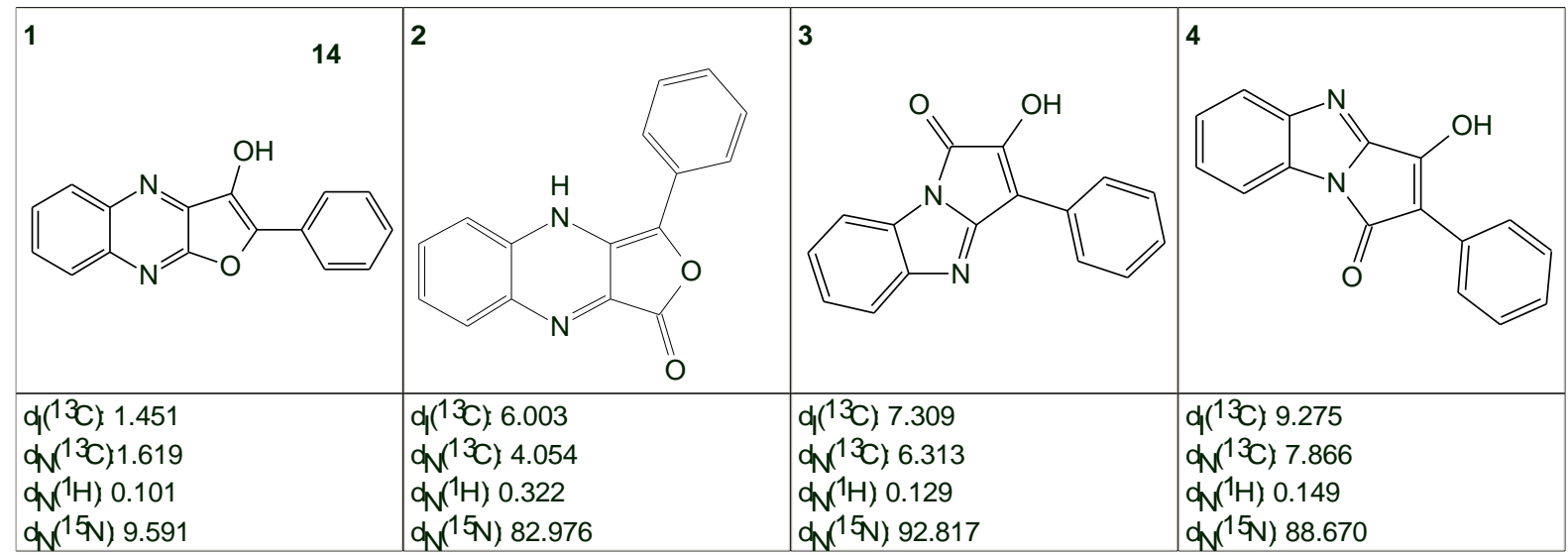

Figure 7. Output structural file ranked by $d_{N}\left({ }^{13} \mathrm{C}\right)$ deviation.

Structure $\mathbf{1 4}$ is the best structure according to the shift predictions for all nuclei presented in Figure 7. Moreover, the deviations for structure $\mathbf{1 4}$ are dramatically smaller than those for the next (\#2) ranked structure for all nuclei and suggests a high reliability for the solution ${ }^{[18]}$. Note that the $d_{N}\left({ }^{13} \mathrm{C}\right)$ deviation for structure 14 is almost 4 times smaller than the average deviation calculated by the GIAO approach. Figure 8 shows the chemical shift assignments performed for structure $\mathrm{C}$ and deduced by the authors ${ }^{[22]}$ and listed with deviations calculated by us. 


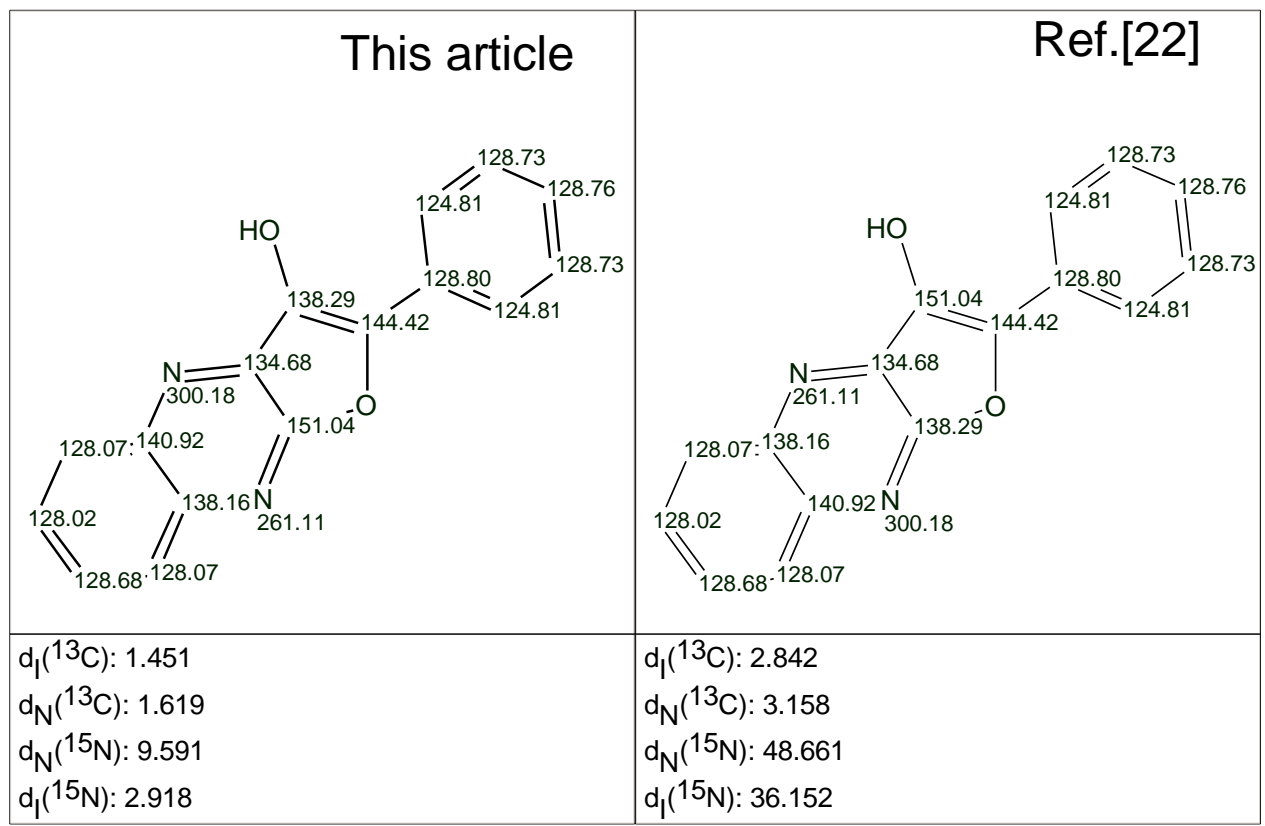

Figure 8 . The chemical shift assignments for structure 14 automatically determined in this article and deduced by the original article authors ${ }^{[22]}$. The deviation values indicate that some chemical shifts assigned on the basis of QM prediction should be exchanged.

All deviation values calculated for the new assignment (including the deviations $d\left({ }^{15} \mathrm{~N}\right)$ ) are smaller than those found for the former assignment. The largest errors are related to the carbons at 151.4 and $138.29 \mathrm{ppm}$, differences of 11 and $16.5 \mathrm{ppm}$. It is also interesting to note that all suggested structures 12-17 except structure 14 were not generated by the program since the Atom Property Correlation Table (APCT) prevents the assembly of structures whose atoms would have chemical shifts differing dramatically from the experimental shifts.

For completeness we repeated structure generation with both the APCT and Filters (structural and spectral) switched off. Finally, 59 non-isomorphic structures were generated including structures 12 and 13 characterized by ${ }^{13} \mathrm{C}$ chemical shift deviations of 6-8 ppm. Structures 15 and 17 could not be generated because according to the initially postulated conditions the mono-substituted benzene ring is connected to the carbon atom with a shift of 144.42 $\mathrm{ppm}$ whose hybridization was assigned as $\mathrm{sp}^{2}\left(\mathrm{sp}^{3}\right.$ is necessary for $\mathbf{1 5}$ and $\left.\mathbf{1 7}\right)$. 
Using the ${ }^{13} \mathrm{C}$ chemical shift values predicted for structure 14 by DFT and NN methods we calculated linear regressions for the experimental shift values based on our shift assignments relative to the assignments suggested by the authors ${ }^{[22]}$, Figure 9.

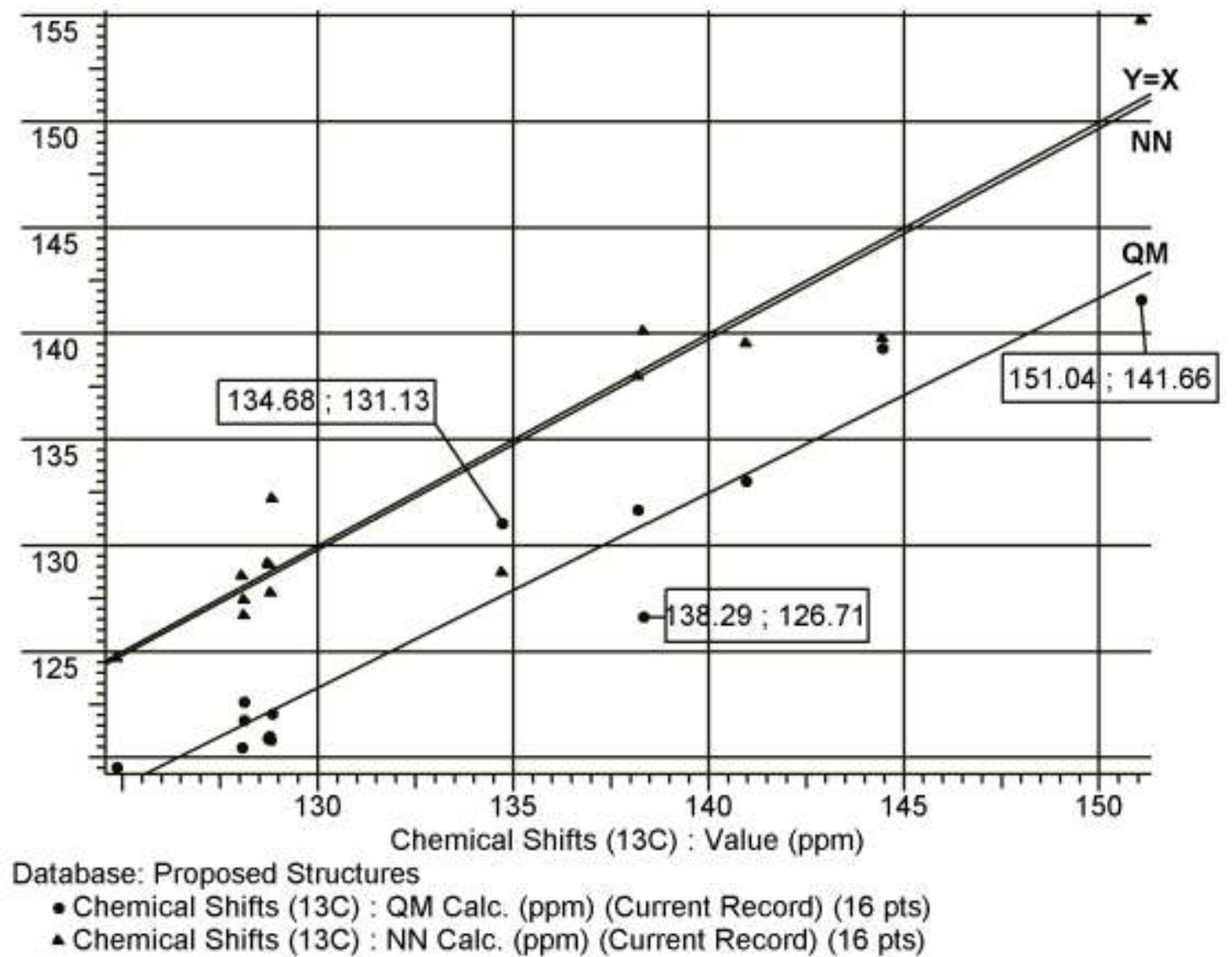

Figure 9. Correlation plots of ${ }^{13} \mathrm{C}$ chemical shift values predicted for structure $\mathbf{1 4}$ (our assignment) by the DFT and NN methods versus the experimental shift values. The target line $\mathrm{Y}=\mathrm{X}$ is shown. The coordinates of some points are shown in frames where the first value designates the experimental shift and the second represents the calculated shift. The regression parameters are: $\mathrm{R}^{2}(\mathrm{NN})=0.905, \mathrm{R}^{2}(\mathrm{QM})=0.937, \mathrm{rms}(\mathrm{NN})=2.54 \mathrm{ppm}$ and $\mathrm{rms}(\mathrm{QM})=1.89 \mathrm{ppm}$. 


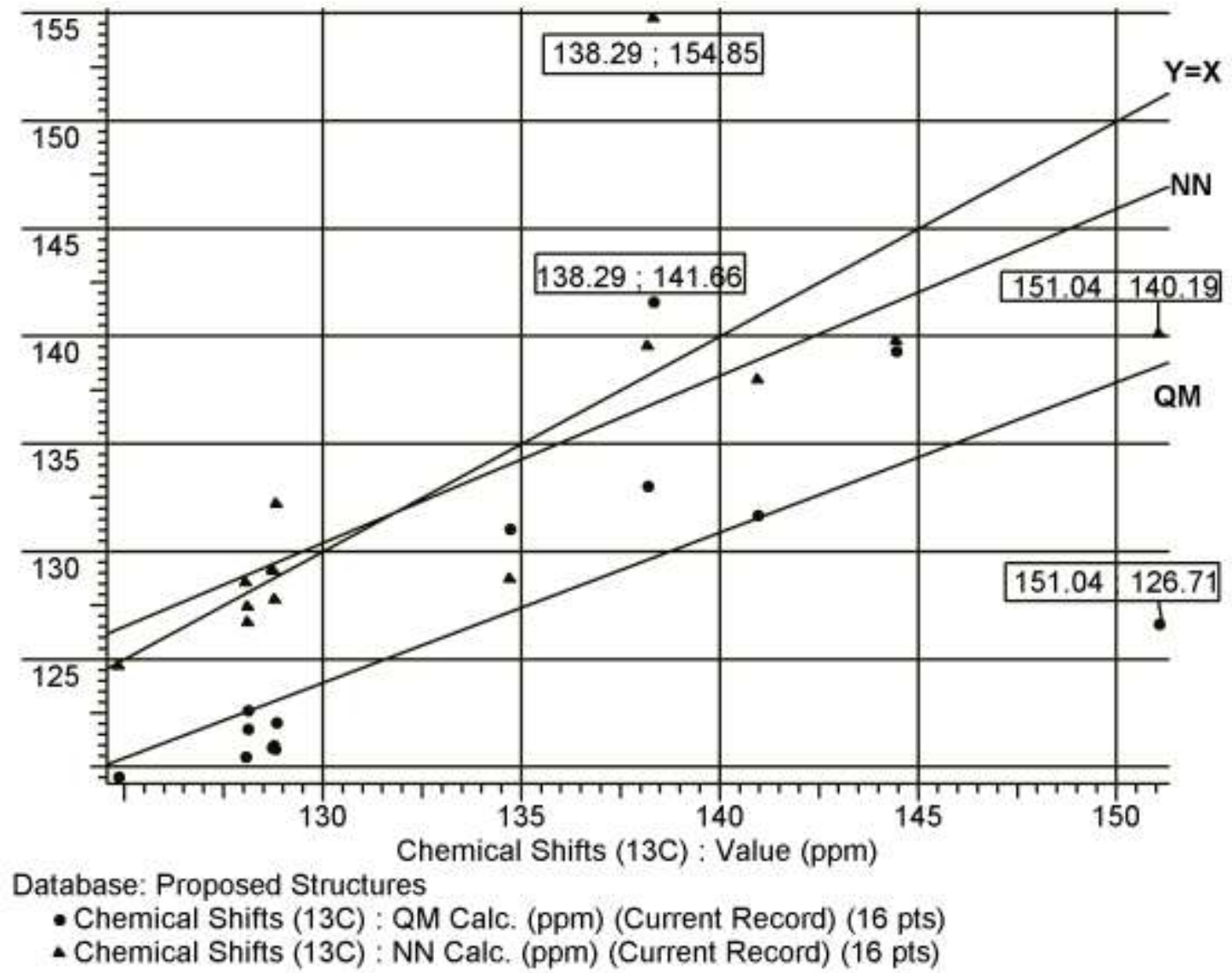

Figure 10. The correlation plots of the ${ }^{13} \mathrm{C}$ chemical shift values predicted for structure 14 (assignment given in reference ${ }^{[22]}$ ) by DFT and NN methods versus the experimental shift values. The target line $\mathrm{Y}=\mathrm{X}$ is shown. The coordinates of some points are shown in frames where the first value designates the experimental shift and the second represents the calculated value. The regression parameters are $\mathrm{R}^{2}(\mathrm{NN})=0.551, \mathrm{R}^{2}(\mathrm{QM})=0.538, \mathrm{rms}(\mathrm{NN})=5.54 \mathrm{ppm}$ and $\operatorname{rms}(\mathrm{QM})=5.10 \mathrm{ppm}$.

Figure 9 shows that for chemical shifts calculated by the NN algorithm the regression line is very close to the $\mathrm{Y}=\mathrm{X}$ line. The DFT line lies $7 \mathrm{ppm}$ below the $\mathrm{Y}=\mathrm{X}$ line. We can observe that the value of $\mathrm{R}^{2}(\mathrm{DFT})$ suggests only an acceptable linear correlation between the experimental and predicted shifts. However, it does not characterize the true quality of prediction. Consequently, the $\mathrm{R}^{2}$ criterion should not be considered as a measure of intrinsic prediction precision for a given method. As our experience shows the average deviation is an effective and rather reliable criterion for selection of the most probable structure. The difference between the DFT- and NNregression lines can be accounted for systematic errors in the calculations performed with the 
GIAO approximation. These errors may be different for different molecules, different versions of the DFT based programs and for different ranges of ${ }^{13} \mathrm{C}$ NMR spectrum. Figure 10 confirms the conclusion regarding the chemical shift assignment given in reference ${ }^{[22]}$ : both $\mathrm{NN}$ and QM chemical shift predictions indicate that the assignment is incorrect. As the article does not contain an atom numbering scheme maybe some confusion in the carbon atom labeling arose when spectral information was listed in the article $\mathrm{e}^{[22]}$.

Since the authors ${ }^{[22]}$ do not report how the ${ }^{13} \mathrm{C}$ experimental chemical shifts were assigned to the carbons of all proposed structures 12-17 (except structure 14) it was not possible to calculate the linear regressions for the $\mathrm{NN}$-calculated shifts for all supposed structures.

Therefore, we predicted the ${ }^{13} \mathrm{C}$ NMR chemical shifts for structures A-F and then graphically compared the predicted spectra with the experimental one as shown in Figure 11.

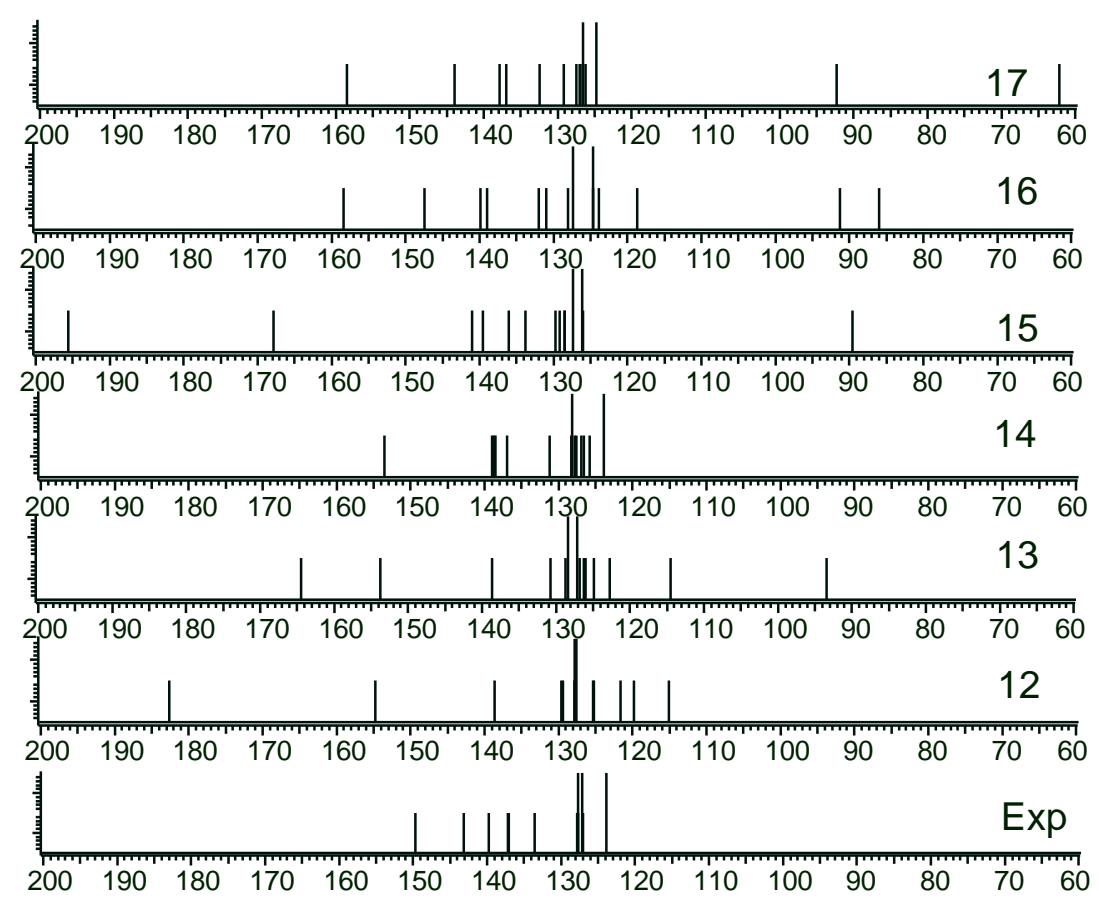

Figure 11. Experimental ${ }^{13} \mathrm{C}$ chemical shifts in comparison with the chemical shifts predicted by the neural net $(\mathrm{NN})$ algorithm for proposed structures 12-17.

The difference between the experimental and NN-predicted spectra is dramatic for all structures except structure 14. All incorrect structures could be immediately rejected before performing 
QM calculations and the right structure would be quickly identified if hypotheses were offered by a human expert.

The authors ${ }^{[22]}$ note that "an attempt to predict ${ }^{1} \mathrm{H}$ and ${ }^{13} \mathrm{C}$ NMR shifts values of structure $\mathbf{1 4}$ based on additivity rules... would be totally unsuccessful" because.. " estimation of chemical shifts according to the additive scheme implemented in the "estimate" utility of CambridgeSoft's ChemDraw Program ${ }^{[33]}$ gives very poor prediction of ${ }^{13} \mathrm{C}$ chemical shifts". With a more appropriate prediction algorithm ${ }^{[8,9,34-36]}$, this comment is obviously invalid.

We share the authors' enthusiasm regarding progress in QM methods applied to chemical shift prediction. With our results described above however the conclusion that "non-empirical calculations of chemical shifts are very cheap in the sense of computational costs and most of the researchers can run them easily on their desk computers (3-5 h per one isomer on a Pentium 4 2.8 $\mathrm{GHz}$ processor with $512 \mathrm{MB}$ RAM) provides a less than ideal situation relative to the use of other computational approaches for NMR prediction.

Example 2.

The same group of authors ${ }^{[32]}$ reported the application of a more complex molecule structure elucidation strategy that was described in their work ${ }^{[22]}$. A novel organic compound was investigated by 1D and 2D NMR experiments (DEPT, NOESY, COSY, HSQC, HMBC, and HMBC ${ }^{1} \mathrm{H}-{ }^{15} \mathrm{~N}$ ). El mass spectra were recorded on a TRACE MS instrument (Finnigan MAT) and a MALDI mass spectrum was also obtained. From the MS data a molecular formula of $\mathrm{C}_{27} \mathrm{H}_{22} \mathrm{~N}_{4} \mathrm{O}_{3}$ was established. 2D NMR data analysis allowed the authors to assemble three fragments with chemical shifts assigned to ${ }^{1} \mathrm{H},{ }^{13} \mathrm{C}$ and ${ }^{15} \mathrm{~N}$ nuclei. Two quaternary $\mathrm{C}$ atoms, one $\mathrm{N}$ atom, and three $\mathrm{O}$ atoms remained unassigned.

The authors ${ }^{[32]}$ suggested two structural hypotheses (19 and 20) which are both in accordance with the molecular formula $\mathrm{C}_{27} \mathrm{H}_{22} \mathrm{~N}_{4} \mathrm{O}_{3}$ and four other structures (15-18) with a molecular formulae differing from that determined from the MS data (Figure 12): 

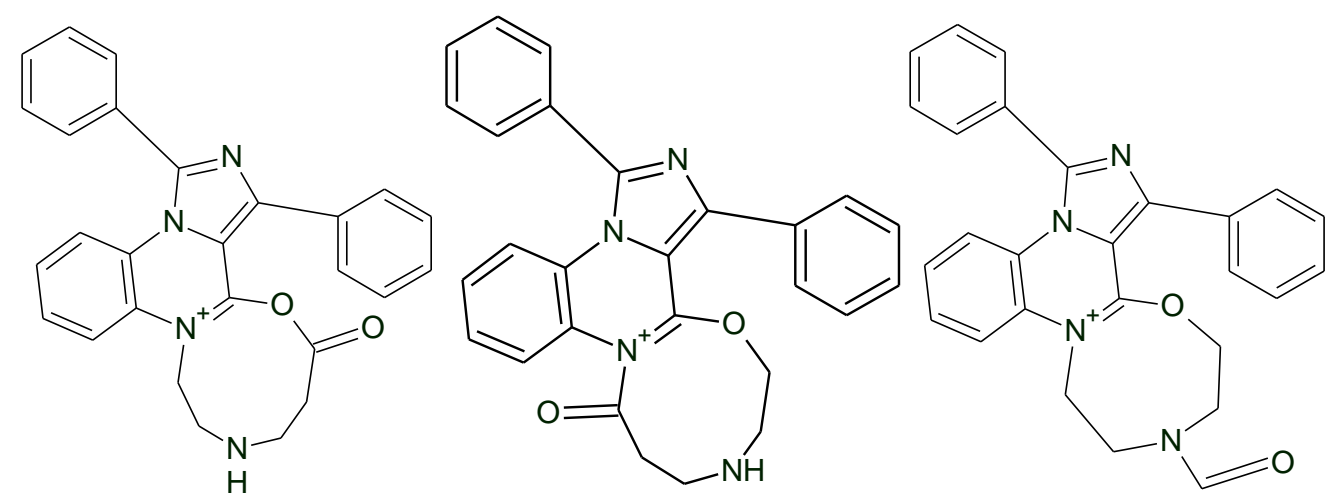

18: $\mathrm{C}_{27} \mathrm{H}_{23} \mathrm{~N}_{4} \mathrm{O}_{2}$<smiles>O=C1C(=O)C23OCCN(CCN12)c1ccccc1-n1c(-c2ccccc2)nc(-c2ccccc2)c13</smiles><smiles>O=C1OCCN1CCOc1nc2ccccc2n2c(-c3ccccc3)nc(-c3ccccc3)c12</smiles>

$20: \mathrm{C}_{27} \mathrm{H}_{23} \mathrm{~N}_{4} \mathrm{O}_{2}$<smiles>O=C1OCCN1CCn1c(=O)c2c(-c3ccccc3)nc(-c3ccccc3)n2c2ccccc21</smiles>

21: $\mathrm{C}_{27} \mathrm{H}_{22} \mathrm{~N}_{4} \mathrm{O}_{2}$

22: $\mathrm{C}_{27} \mathrm{H}_{22} \mathrm{~N}_{4} \mathrm{O}_{3}$

23: $\mathrm{C}_{27} \mathrm{H}_{22} \mathrm{~N}_{4} \mathrm{O}_{3}$

Figure 12. Structures suggested in the work $^{[32]}$.

To identify the best structure from among the suggestions $\mathbf{1 8 - 2 3}$, Balandina et al calculated the

${ }^{13} \mathrm{C}$ chemical shifts for all candidate structures using QM methods. As a result of statistical processing of the data, structure $\mathbf{2 3}$ was selected as the most preferable structure since its correlation coefficient of $\mathrm{R}=0.996$ was the highest in value and the $\mathrm{MAD}=5.65 \mathrm{ppm}$ was the minimum.

We attempted to solve this problem by employing the usual strategy for the StrucEluc system to elucidate unknown structures using the Fragment Mode. On the basis of spectral data presented in the article ${ }^{[32]}$, the program produced the MCD shown in Figure 13 

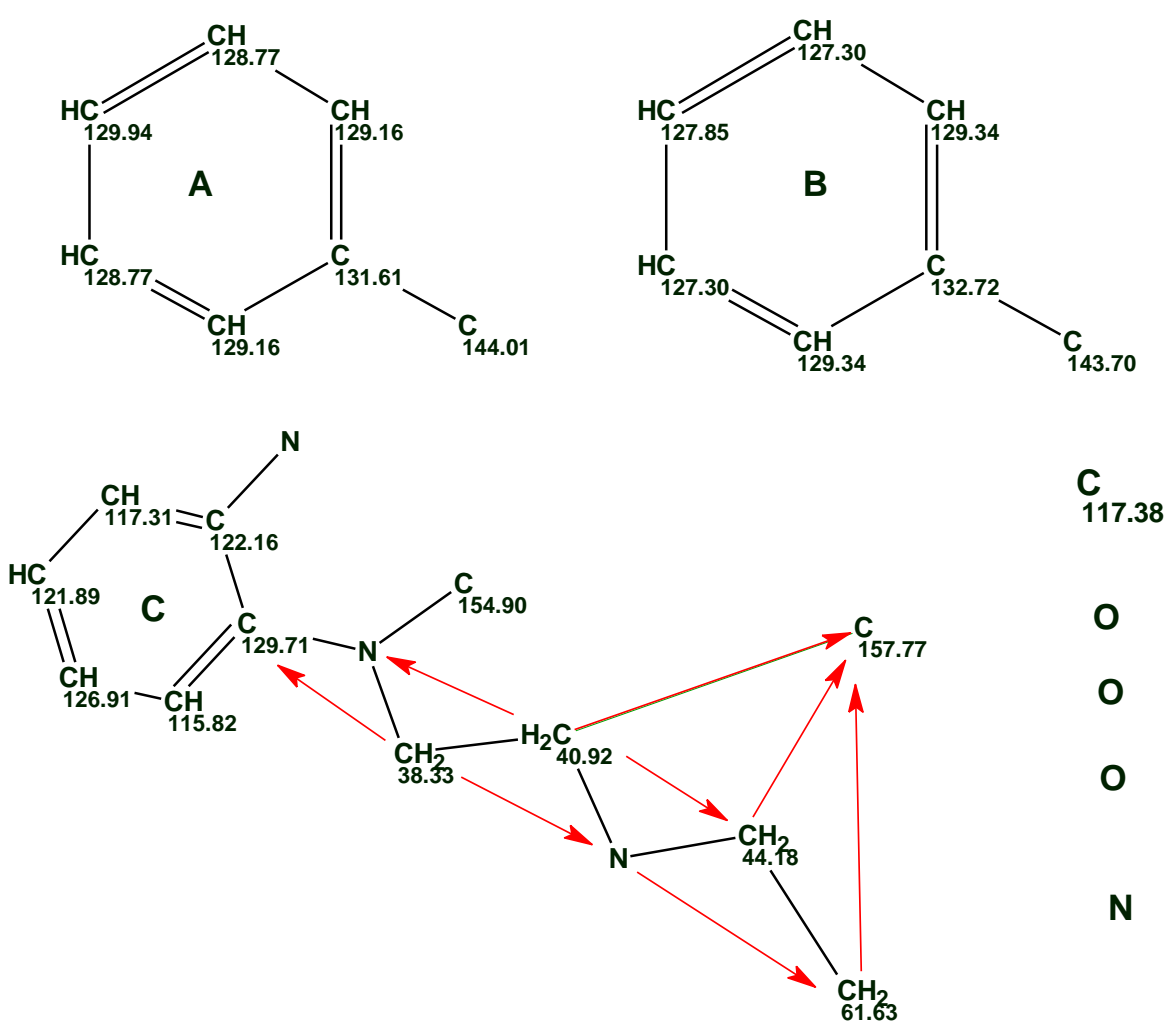

Figure 13. The molecular connectivity diagram containing the three fragments derived by Balandina et $\mathrm{al}^{[32]}$. The red arrows denote HMBC correlations.

The atom corresponding to the chemical shift of 157.77 ppm was introduced with the property $s p^{2} /$ not defined, while the atom corresponding to $117.38 \mathrm{ppm}$ was assigned the property of not defined/not defined as the indicated chemical shift can be observed either for $s p^{2}$ or $s p^{3}$ (O-C-O) hybridized carbons. HMBC connectivities are marked by red lines in Figure 13.

Structure generation accompanied by Structural Filtering was performed under standard conditions. To reduce the output file, 3- and 4-membered cycles were forbidden and a Geometry option was enabled to exclude deliberately "ugly" structures. The results gave: $411 \rightarrow 44 \rightarrow 25$, $t_{g}=0.9 \mathrm{~s}$. For all structures, ${ }^{13} \mathrm{C}$ chemical shifts were calculated by $\mathrm{NN}$ and incremental approaches. The first three structures of the ranked file are shown in Figure 14. 


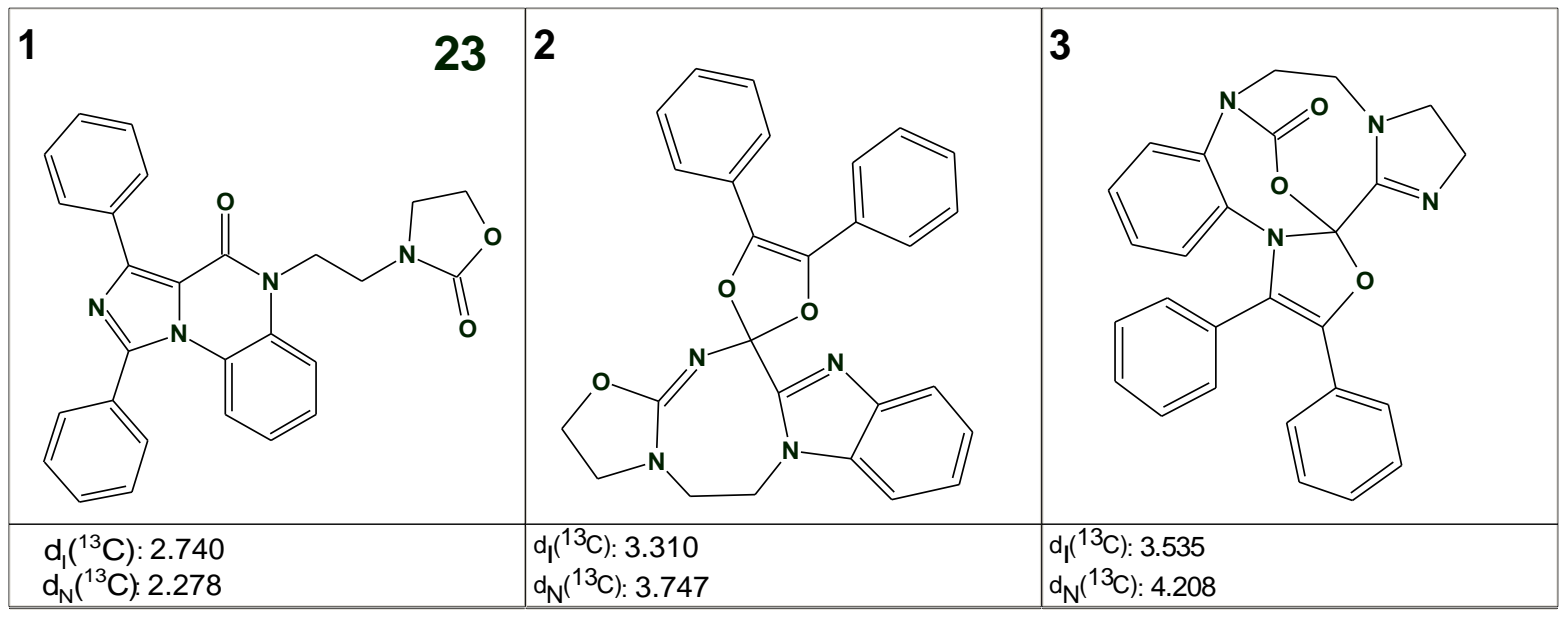

Figure 14 . The output structural file ranked by $d_{N}\left({ }^{13} \mathrm{C}\right)$ deviation.

Comparison of the best structure \#1 of the ranked file with the suggested structures establishes structure \#1 as identical to structure $\mathbf{2 3}$. Therefore structure $\mathbf{2 3}$ corresponds to the molecular formula and the constraints graphically represented in the MCD. Structure $\mathbf{2 3}$ and the largest fragment $\mathbf{C}$ depicted in the $\mathrm{MCD}$ contains a chain $-\mathrm{N}-\mathrm{CH}_{2} \mathrm{CH}_{2}-\mathrm{N}-$, while this fragment is absent from the structure 22. Structure $\mathbf{2 2}$ is possible only if the initial set of constraints is changed. In this case it would mean that the chain part of the large fragment which is well confirmed by ${ }^{1} \mathrm{H}$ ${ }^{13} \mathrm{C}$ and ${ }^{1} \mathrm{H}-{ }^{15} \mathrm{~N}$ HMBC correlations has been determined incorrectly. There is however no grounds for revision of the mentioned chain.

For structures 18-21 all have molecular formulae differing from that determined experimentally. Though possible it is unlikely. In addition structure 19 contains a fragment that contradicts the side chain of the core fragment $\mathbf{C}$ discussed previously. Structure $\mathbf{2 0}$ would show a doublet signal for the carbonyl group and, consequently, can be rejected for this reason. Finally, the carbon atom at $154.9 \mathrm{ppm}$ in structure $\mathbf{2 1}$ has to be $s p^{3}$ hybridized and this is impossible. The structural suggestions are therefore not appropriate. The authors ${ }^{[32]}$ nevertheless discussed all six structures as part of their methodological analysis in the publication. 
Since structures $18\left(\mathrm{C}_{27} \mathrm{H}_{23} \mathrm{~N}_{4} \mathrm{O}_{2}\right)$ and $21\left(\mathrm{C}_{27} \mathrm{H}_{22} \mathrm{~N}_{4} \mathrm{O}_{2}\right)$ contain fragment $\mathbf{C}$ we performed the structure elucidation process using these molecular formulae to obtain all consequences of replacing these constraints. New MCDs were created and the following results were obtained:

\section{$\underline{\text { Structure } 18}$}

$k=88, t_{g}=2.5 \mathrm{~s}$, with structure 18 selected as the best with $d_{N}\left({ }^{13} \mathrm{C}\right)=4.24 \mathrm{ppm}$. Structure 18 should be rejected as the deviation is almost twice as large as that obtained for structure $\mathbf{2 3}$.

\section{$\underline{\text { Structure } 21}$}

Fuzzy Structure Generation was run with the APCT and Spectral Filters switched off since structure $\mathbf{2 1}$ would not be generated with these filters switched on.

Result: $k=13826 \rightarrow 13569 \rightarrow 271, t_{g}=61 \mathrm{~s} .{ }^{13} \mathrm{C}$ chemical shifts for the resulting 13569 structures were calculated using the incremental method in 77 s. Structure 21 was again selected as the best structure with $d_{N}\left({ }^{13} \mathrm{C}\right)=4.81 \mathrm{ppm}$ and $d_{I}\left({ }^{13} \mathrm{C}\right)=6.07 \mathrm{ppm}$. The deviation values are large enough to suggest that, in this case, structure $\mathbf{2 1}$ has been "forcibly" derived from the available NMR experimental data. It is worthy to note that the predicted chemical shift for the carbon atom at $154.90 \mathrm{ppm}$ differs from the experimental value by $>70 \mathrm{ppm}$.

Balandina et al again compared their results with NMR chemical shift prediction contained within the Cambridgesoft ChemOffice program. They stated that "information on the chemical shifts for the carbon atom of the $\mathrm{N}^{+}=\mathrm{C}-\mathrm{O}$ fragment is lacking in the database of ChemOffice program package... Hence, if these structures were formed in the course of the reaction, they could not be established in terms of this empirical approach". Based on the data obtained for structure $\mathbf{1 8}$ the current study showed that this limitation was not present in our program $^{[8,15]}$. In spite of the fact that the examples employed for justification of a methodology based on QM NMR calculations seems to be rather weak, we agree with authors ${ }^{[32]}$ conclusion that the "combined use of modern 2D NMR experiments and ab initio chemical shift calculations is efficient". This approach may be the only computational approach if a molecule contains exotic substructures that are unknown for a program based on empirical methods of spectrum 
prediction. At the same time CASE expert systems supplied with fast and accurate algorithms for empirical chemical shift prediction can frequently help the researcher to avoid time consuming QM computations.

\section{Selection of the correct structure without using a CASE application.}

As shown in previous sections the application of a CASE expert system allows one to obtain a full set of possible structures for which all experimental ${ }^{13} \mathrm{C}$ and ${ }^{1} \mathrm{H}$ chemical shifts are assigned to the atoms composing the structures. Nevertheless, there are situations when 2D NMR data cannot unambiguously solve a problem and it is necessary to make a choice between isomers of very similar structures. In such situations it is obviously desirable to find the best candidate structure without additional experimentation, for example X-ray analysis. NMR spectrum prediction can be attractive for this purpose. So, again the question arises: are modern empirical methods of chemical shift prediction capable of delivering a solution with appropriate confidence? Here we consider several examples where a solution to a problem was found using both QM and empirical predictions applied independently.

Example 1.

The structures of two new isomeric quinoxalines, $\mathbf{2 4}$ and $\mathbf{2 5}$, with molecular formula $\mathrm{C}_{21} \mathrm{H}_{13} \mathrm{~N}_{5} \mathrm{O}_{2}$ were established in the reported work ${ }^{[31]}$ :

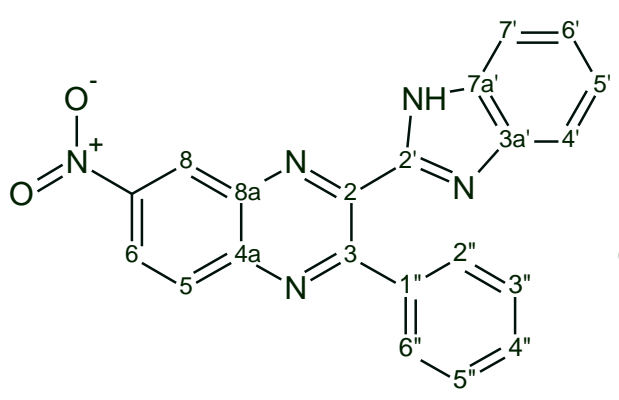

24

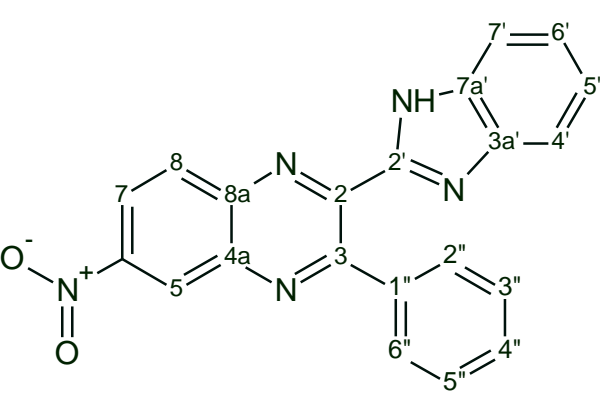

25

Both isomers had almost identical ${ }^{1} \mathrm{H}$ NMR spectra but different ${ }^{13} \mathrm{C}$ spectra. In order to determine the correspondence between the spectral data and the structures the authors used ${ }^{13} \mathrm{C}$ NMR chemical shift prediction using the DFT method. The authors suggested that the spectra 
could be attributed to the corresponding structures ( $\mathbf{2 4}$ or $\mathbf{2 5}$ ) by assuming mutual trends in the calculated and experimental shifts for atoms which are most sensitive to the position of the $-\mathrm{NO}_{2}$ group. In this case atoms C-2, C-3, C-4a and C-8a were allocated as the most sensitive. The trend was discovered by comparing the ${ }^{13} \mathrm{C}$ chemical shifts calculated by the QM methods with the experimental shifts of the corresponding pairs of atoms. As a result the experimental spectrum of substance 1 was related to isomer 25 and spectrum 2 to isomer 24.

The authors stated: "If the molecular fragments are simple and linked by one bond, empirical chemical shift increments can be exploited to predict the influence of neighboring groups and in this way to establish the overall structure. However, there are many cases where fragments are bonded by two or even three bonds. In such cases simple empirical rules cannot be deduced to take into account the influence of the vicinal fragments." We agree that simple empirical rules can be challenging to apply successfully for the prediction of accurate chemical shifts in such highly conjugated systems such as isomers $\mathbf{2 4}$ and $\mathbf{2 5}$. However, in the process of developing our algorithms ${ }^{[13,15]}$ we placed high emphasis on taking into account the effects of conjugation. Therefore the particular problem discussed here was used by us as an example to challenge our program. We attempted to establish a one-to-one correspondence between the structures and spectra using ${ }^{13} \mathrm{C}$ chemical shift prediction performed using our neural net algorithm.

For the ${ }^{13} \mathrm{C}$ spectra of substances 1 and 2 we compared the four experimental chemical shifts related by the authors ${ }^{[31]}$ to the four "representative" atoms $(\mathrm{C}-2, \mathrm{C}-3, \mathrm{C}-4 \mathrm{a}$ and C-8a) in both isomers. These chemical shifts were compared with those calculated by QM and NN approaches. The initial data are presented in Table 1.

\section{Table 1.}

Using these data we calculated the $R^{2}$ and standard errors (SE) for all 8 combinations of spectra and structures. In other words we checked how each of the spectra 1 and 2 correlates with structures $\mathbf{2 4}$ and $\mathbf{2 5}$. The results are shown in Table 2.

\section{Table 2.}


The table shows that according to both methods of spectrum prediction, the chemical shifts of isomer $\mathbf{2 4}$ more stronger correlate with spectrum 2, while the chemical shifts of isomer $\mathbf{2 5}$ correlate with spectrum 1 . This agrees with the conclusion made by the authors ${ }^{[31]}$. Figure 15 demonstrates that the most important chemical shifts calculated using the NN approach are closer to the experimental values that those obtained by the QM calculations, while both approaches are capable of revealing the general trend.

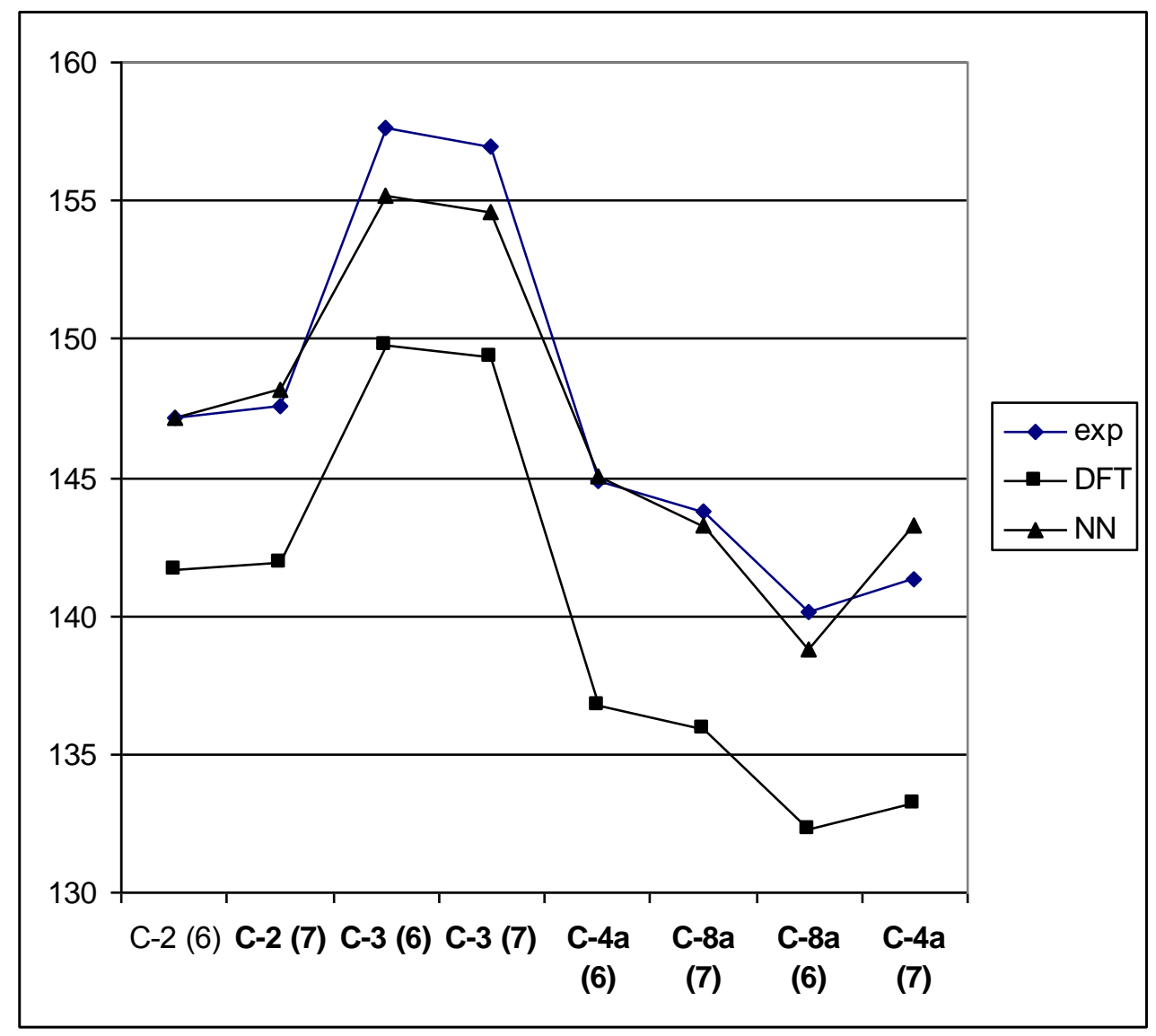

Figure 15. A comparison of the experimental chemical shifts with those calculated for selected atoms using the $\mathrm{NN}$ and QM approaches. The numbers in brackets correspond to the structure numbers.

Figures 16 and 17 allow us to compare the accuracy of chemical shift prediction performed by both methods. It is evident that the precision of chemical shift prediction by the NN method provides the possibility to utilize calculated ${ }^{13} \mathrm{C}$ NMR spectra of large enough conjugated molecules which contain a significant number of heteroatoms. It should be noted that the neural net prediction of ${ }^{15} \mathrm{~N}$ chemical shifts suggests that the nitrogen atom in the position meta to the 
$\mathrm{NO}_{2}$ group in isomer 24 will have a chemical shift equal to $\sim 320 \mathrm{ppm}$, while a chemical shift $303 \mathrm{ppm}$ is expected for the nitrogen atom in the para-position. It is predicted that for isomer $\mathbf{2 5}$ both $\mathrm{N}$ atoms would have close chemical shifts near $315 \mathrm{ppm}$. We expect that these spectral features would also be very useful for confirmation of the spectral assignments made for isomers 24 and 25.

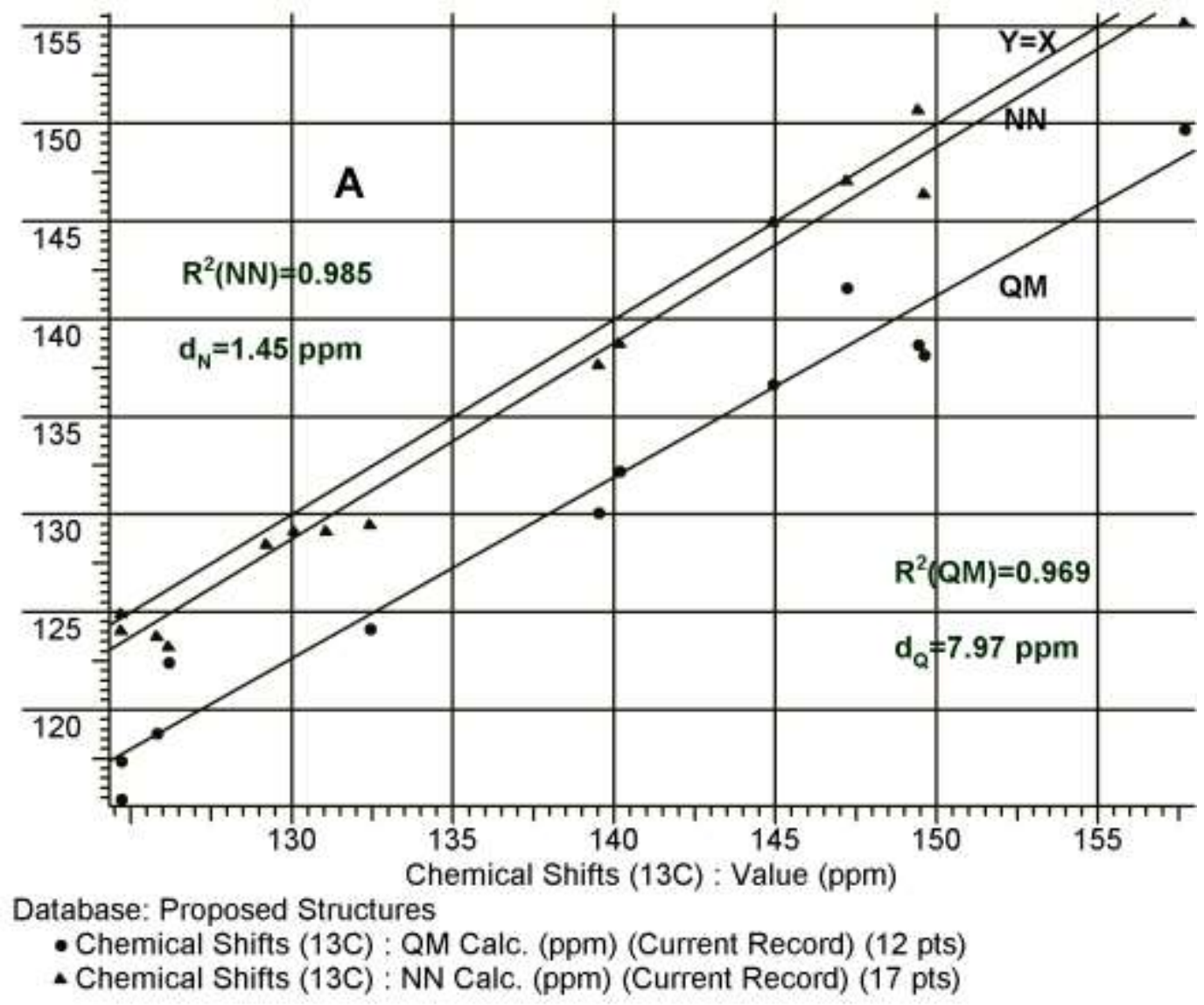

Figure 16. Correlation plots of the ${ }^{13} \mathrm{C}$ chemical shift values predicted for the structure $\mathbf{2 4}$ by DFT (circles) and NN (triangles) methods versus experimental shift values. The target line $\mathrm{Y}=\mathrm{X}$ is shown. 


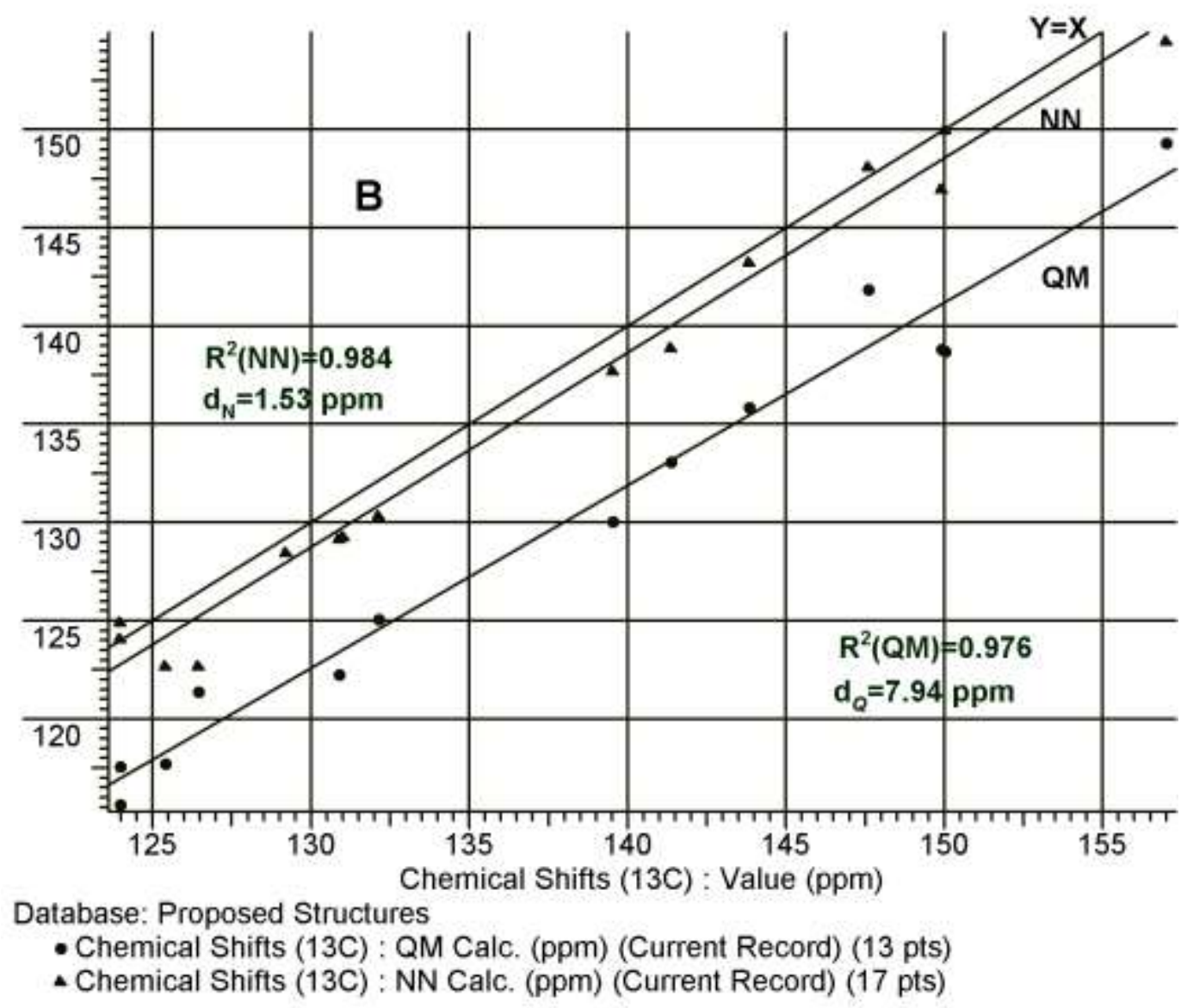

Figure 17. The correlation plots of ${ }^{13} \mathrm{C}$ chemical shift values predicted for structure 25 by DFT (circles) and NN (triangles) methods versus experimental shift values. The target line $\mathrm{Y}=\mathrm{X}$ is shown.

\section{Example 2.}

Barone et al $^{[23]}$ investigated the possibility of structure validation of natural products by the quantum-mechanical GIAO calculations of ${ }^{13} \mathrm{C}$ NMR chemical shifts. We selected two examples given in this work where the analyzed structures are absent from the ACD/Labs database and consequently the results of empirical NMR spectrum prediction were not influenced.

Different groups of researchers suggested the following structures and chemical shift assignments for a natural product magnolialide: 


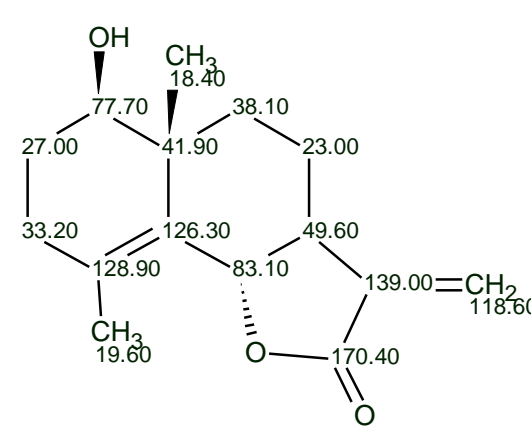

26

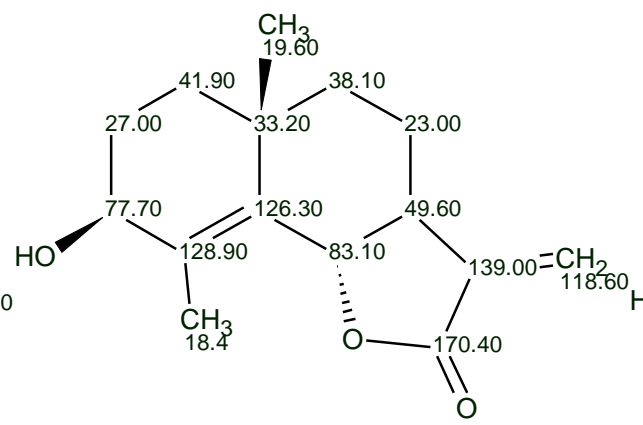

27

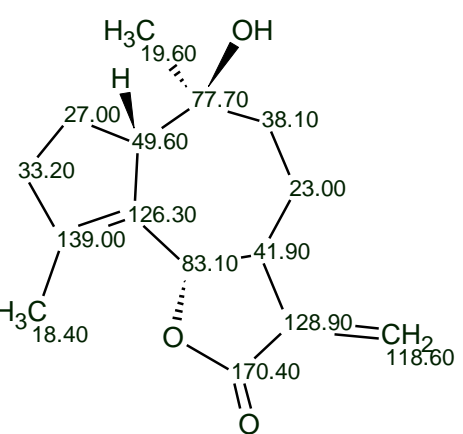

28

It was experimentally established that structure $\mathbf{2 6}$ was the correct structure. GIAO-based chemical shift calculation allowed authors ${ }^{[23]}$ to confidently reject structure $\mathbf{2 8}$. In spite of the similarity in plots showing the differences between experimental and predicted shifts for structures $\mathbf{2 6}$ and $\mathbf{2 7}$ it was found that these differences were slightly larger for $\mathbf{2 7}$. This observation, together with the correlation coefficient, led to the assignment of structure $\mathbf{2 6}$ to magnolialide. In the article ${ }^{[23]}$, all calculations were performed with and without chemical shift scaling, so the results can be compared.

The authors noted that they could discriminate between and assign the chemical shift values $\delta$ $=128.90$ and $126.30 \mathrm{ppm}$ for two of the carbon nuclei which had not been unequivocally assigned previously. The scaled (29) and not scaled (30) chemical shifts of structure 26 are shown below:

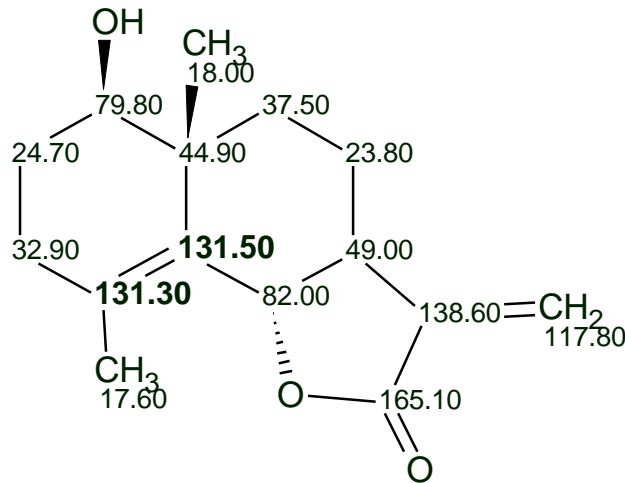

29

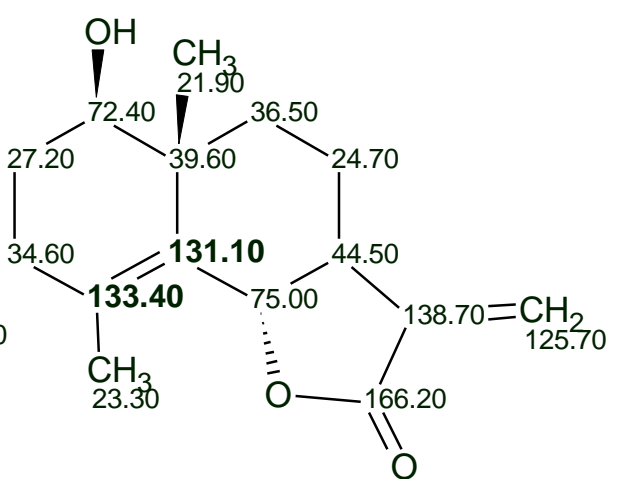

30

We see that the scaled chemical shifts for the corresponding atoms are almost the same (29, 131.3 and 131.5, bold), while there is a difference for the non-scaled set of chemical shifts (30, 133.4 and 131.1, bold). The problem concerning which chemical shifts should be used for 
checking assignments likely careful investigation.

The results of ${ }^{13} \mathrm{C}$ chemical shift prediction for structures $\mathbf{2 6 - 2 8}$ by NN and DFT approaches are shown in Table 3, as well as in Figures 7S-9S (see Supporting Information) where plots are computed for both methods of prediction. For comparison scaled chemical shifts were used as these are closer to the experimental values.

Table 3. Table 3 and Figures 7S-9S show that both methods provide almost the same accuracy for chemical shift prediction. Correct selection of the right structure is provided not only by $\mathrm{R}^{2}$ values, but by the average deviations $d_{N}$ and $d_{Q}$. In accordance with our experiences examining hundreds of structures elucidated using a CASE approach ${ }^{[18]}$, the differences between the average deviations calculated for structures 26-28 allow us to confidently state that structure $\mathbf{2 6}$ is indeed genuine.

Example 3.

Another example considered by Barone et $a l^{[23]}$ is the case when predicted ${ }^{13} \mathrm{C}$ chemical shift values for the correct and incorrect structures are very similar. The structures are shown as $\mathbf{3 1}$, the correct structure, and 32, the incorrect structure.

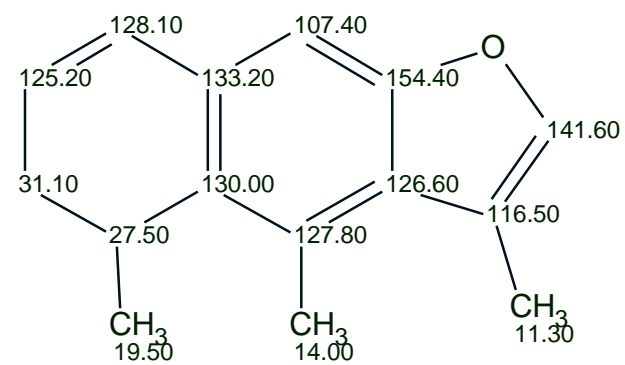

31

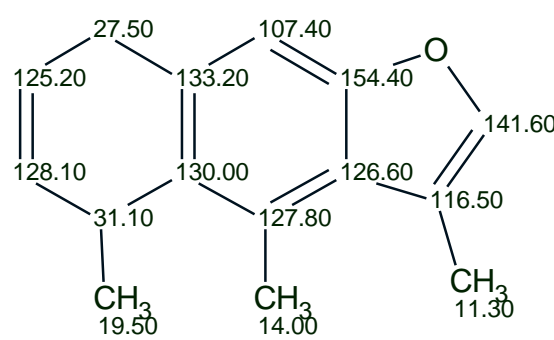

32

The incorrect structure (32) differs from the correct structure (31) only by the position of a double bond. The authors conclude that neither the chemical shift value, nor the $\Delta \delta$ analysis, allow an unambiguous assignment. We were interested to compare the predictions of both QM and $\mathrm{NN}$ approaches for these very similar conjugated structures. The results are presented below and in Figures 10S-11S (Supporting Information): 
Structure 31: $R^{2}(\mathrm{QM})=0.9988, R^{2}(\mathrm{NN})=0.9991, d_{Q}=1.56 \mathrm{ppm}, d_{N}=1.64 \mathrm{ppm}$

Structure 32: $R^{2}(\mathrm{QM})=0.9980, R^{2}(\mathrm{NN})=0.9982, d_{Q}=2.05 \mathrm{ppm}, d_{N}=2.18 \mathrm{ppm}$

The regression plots and parameters characterizing the quality of correlation calculated by both methods are very similar, but both methods indicate the correct structure and again the differences in average deviations $(0.5 \mathrm{ppm})$ for both structures are acceptable in terms of confirming the choice made.

\section{The combined application of empirical and non-empirical methods of chemical shift assignment.}

Bagno and Saielli ${ }^{[21]}$ published an interesting overview of advances in quantum-chemical prediction of NMR spectra and their parameters. As an example of NMR spectrum prediction performed for natural products, they reported a computational study of the molecule of nimbosodione 33.

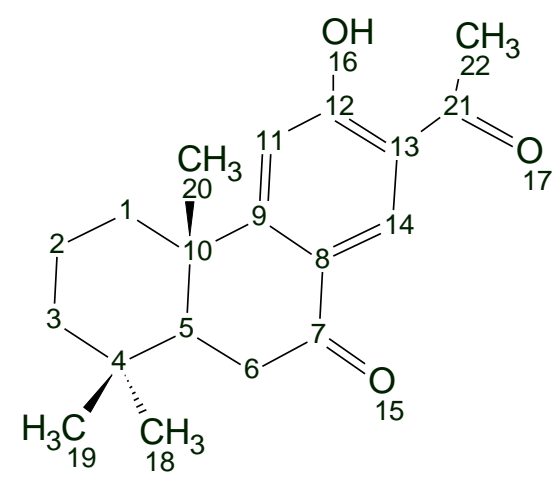

33

Compound 33 was originally isolated and identified by Ara et al ${ }^{[44]}$ from spectral data, but as a result of the total synthesis of nimbosodione and careful analysis of its NMR spectra Li et al ${ }^{[45]}$ showed that the original NMR spectral data and their assignment were incorrect. Even though Li et al ${ }^{[45]}$ measured the ${ }^{1} \mathrm{H}$ and ${ }^{13} \mathrm{C}$ NMR spectra of nimbosodione accurately, they failed to complete a new spectral assignment. Bagno et al ${ }^{[21]}$ calculated the ${ }^{1} \mathrm{H}$ and ${ }^{13} \mathrm{C}$ NMR chemical shifts of structure $\mathbf{3 3}$ using a DFT approach and clearly showed that the spectral assignment and chemical shifts of several definite atoms reported by Ara et al were incorrect. The absence of a 
correct spectral assignment prevented the authors ${ }^{[21]}$ from comparing the predicted and experimental chemical shifts.

In order to allow this we performed a search in the ACD $\backslash$ Labs NMR Database ${ }^{[8]}$ for structures having the framework similar to that of nimbosodione. The database contains more than 290,000 structures with chemical shifts assigned to both carbon and hydrogen atoms. Five structures were found as a result of the search. These structures (ID 2-6) and their assigned ${ }^{13} \mathrm{C}$ chemical shifts are presented in Figure 18. Comparison of the found structures with the nimbosodione structure allowed us to assign the chemical shifts of the compound under investigation (ID 1) as shown in Figure 18. To check the assignment, both the ${ }^{1} \mathrm{H}$ and ${ }^{13} \mathrm{C}$ chemical shifts of nimbosodione were calculated using a neural net approach ${ }^{[15]}$.

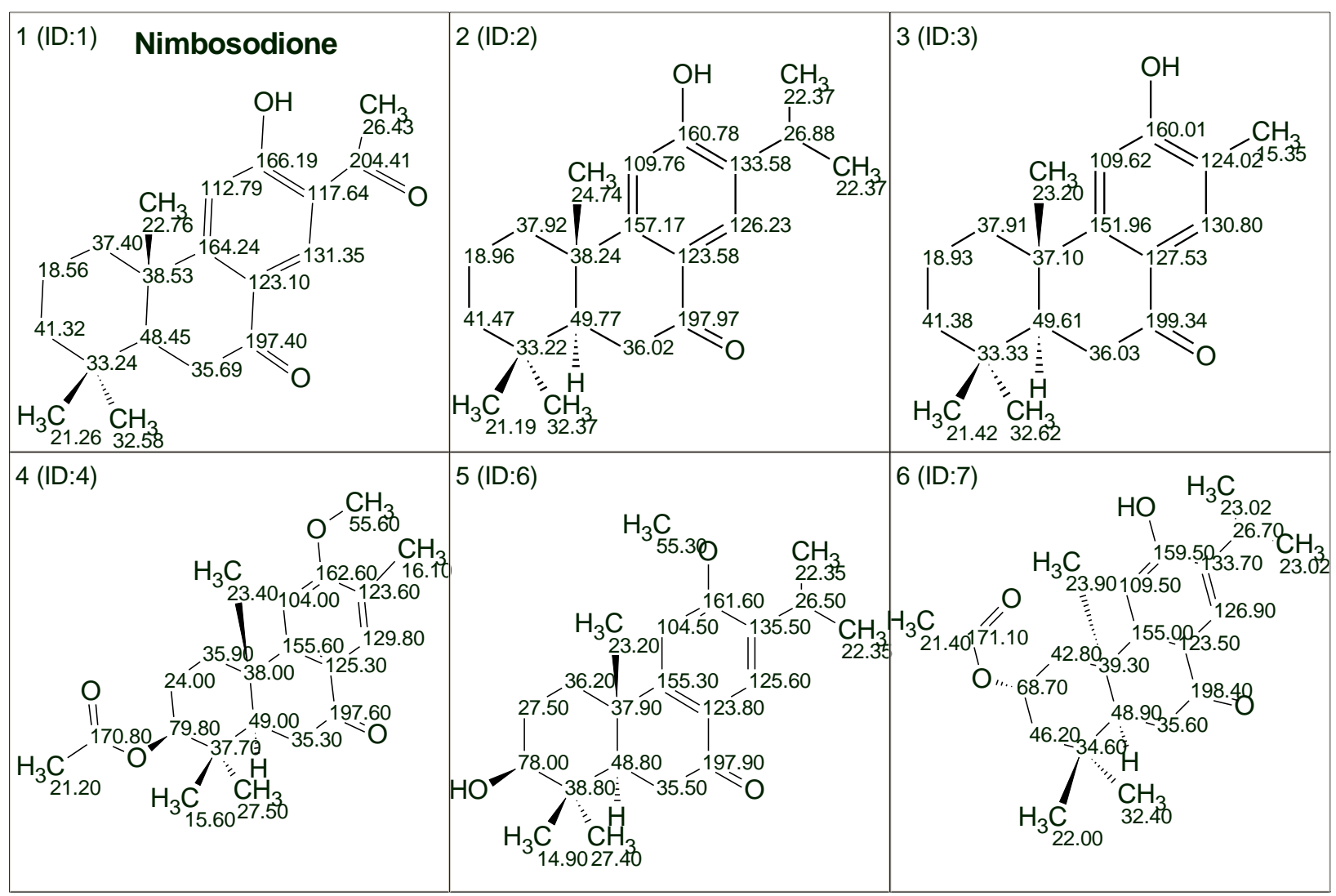

Figure 18. The structure of nimbosodione (ID:1) assigned in accordance with the experimental NMR spectra reported by Li et al ${ }^{[45]}$. The assignments of carbon atoms of the reference structures (ID 2-6) found in the ACD/NMR DB were used.

The two sets of both old and new assigned experimental ${ }^{13} \mathrm{C}$ chemical shifts and the 
corresponding values calculated by DFT and NN approaches are collected in Table 4.

Table 4. A comparison of experimental and calculated ${ }^{13} \mathrm{C}$ chemical shifts for nimbosodione.

\begin{tabular}{|c|c|c|c|c|}
\hline Atom & Exp. $^{[44]}$ & $\begin{array}{c}\text { Exp }^{[45]} \\
\text { our } \\
\text { assignment }\end{array}$ & $\begin{array}{c}\text { Calc. } \\
\text { QM }^{[21]}\end{array}$ & Calc. \\
\hline C1 & 37.95 & 37.4 & 41.75 & 36.8 \\
\hline C2 & 18.90 & 18.56 & 23.78 & 19.38 \\
\hline C3 & 41.37 & 41.32 & 45.41 & 40.92 \\
\hline C4 & 33.31 & 33.24 & 41.19 & 33.87 \\
\hline C5 & 36.03 & 48.45 & 39.53 & 49.38 \\
\hline C6 & 49.58 & 35.69 & 53.63 & 37.25 \\
\hline C7 & 198.60 & 197.4 & 201.75 & 197.77 \\
\hline C8 & 157.08 & 123.1 & 128.25 & 125.46 \\
\hline C9 & 159.08 & 164.24 & 173.26 & 161.31 \\
\hline C10 & 33.31 & 38.53 & 46.34 & 38.89 \\
\hline C11 & 109.62 & 112.79 & 117.47 & 111.48 \\
\hline C12 & 159.15 & 166.19 & 177.21 & 165.79 \\
\hline C13 & 157.42 & 117.64 & 122.09 & 119.57 \\
\hline C14 & 130.78 & 131.35 & 139.49 & 131.98 \\
\hline C18 & 15.10 & 32.58 & 34.35 & 29.17 \\
\hline C19 & 23.22 & 21.26 & 23.06 & 26.49 \\
\hline C20 & 21.31 & 22.76 & 25.07 & 27.53 \\
\hline COCH3 & 32.59 & 26.43 & 28.07 & 29.48 \\
\hline COCH3 & 198.62 & 204.41 & 212.84 & 205.42 \\
\hline
\end{tabular}

Statistical analysis of the data gave the following average chemical shift deviations and $\mathrm{R}^{2}$ values for the old and new assignments:

1) Old assignment $-\mathrm{R}^{2}(\mathrm{DFT})=0.979 ; \mathrm{R}^{2}(\mathrm{NN})=0.981 ; d(\mathrm{DFT})=10.48 \mathrm{ppm}, d(\mathrm{NN})=7.87 \mathrm{ppm}$,

2) New assignment $-\mathrm{R}^{2}(\mathrm{DFT})=0.994 ; d \mathrm{R}^{2}(\mathrm{NN})=0.999 ;(\mathrm{DFT})=6.26 \mathrm{ppm}, d(\mathrm{NN})=1.72 \mathrm{ppm}$,

Both methods of chemical shift calculation provide evidence that the old nimbosodione assignment is incorrect. The old (34) and new (35) measured chemical shifts and their 
assignments are shown on structures $\mathbf{3 4}$ and $\mathbf{3 5}$.

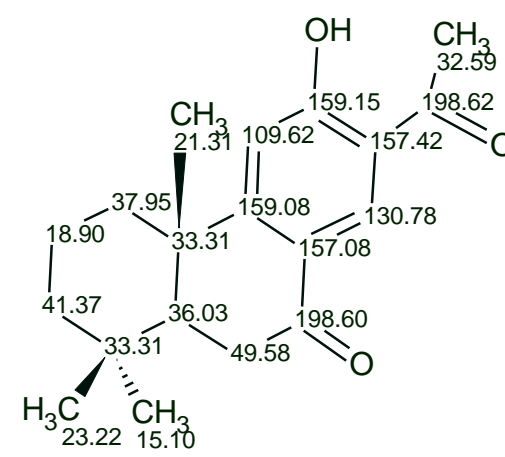

34

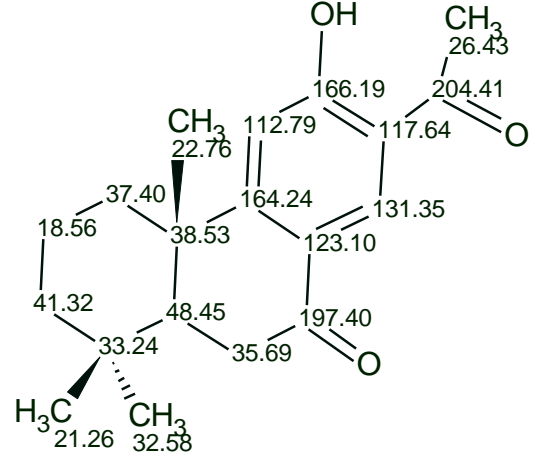

35

The difference between the regression plots calculated for the both new and old assignments can be visually evaluated from Figures 19 and 20. For the old assignment both methods of chemical shift prediction show significant scattering of the calculated chemical shifts and a significant deflection of both plots from the target line. The application of both methods of chemical shift prediction in combination with the data stored in a database containing structures accompanied by their assigned NMR spectra allowed us to confidently determine full chemical shift assignment which was previously impossible. 


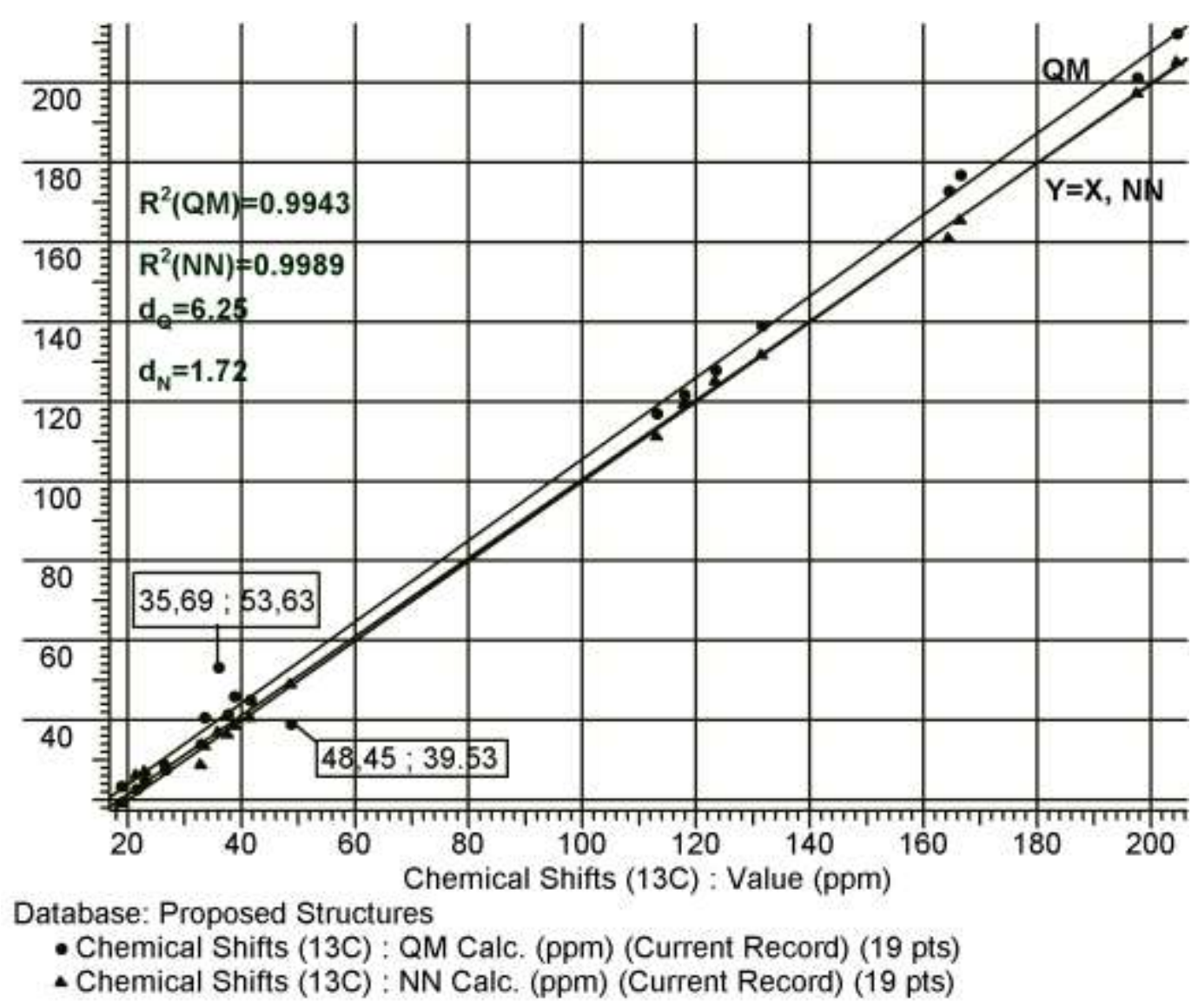

Figure 19. Correlation plots of ${ }^{13} \mathrm{C}$ chemical shift values predicted for the new nimbosodione assignment by DFT (circles) and NN (triangles) methods versus experimental shift values. The $\mathrm{NN}$ regression line almost coincides with the target line $\mathrm{Y}=\mathrm{X}$. 


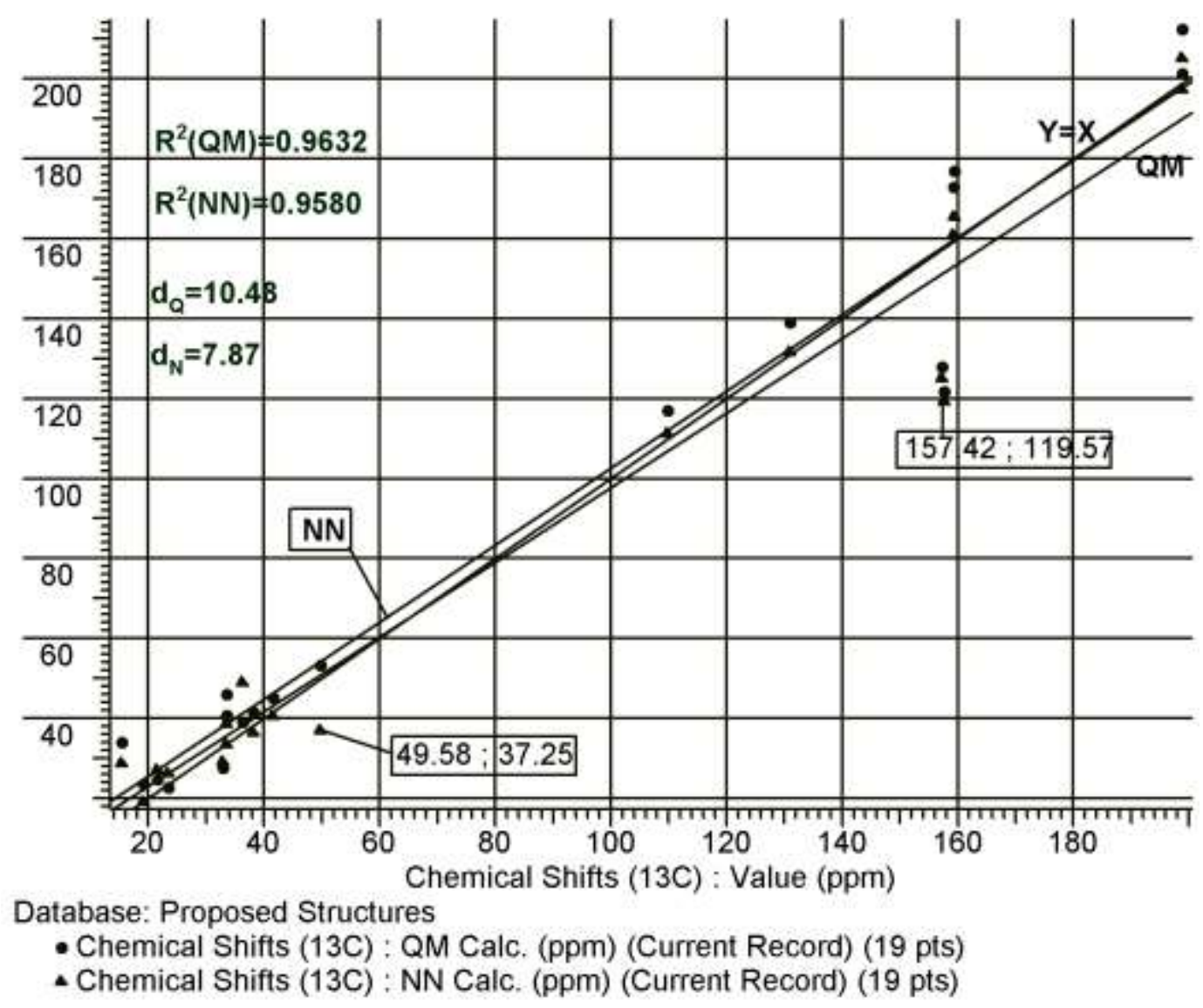

Figure 20. Correlation plots of ${ }^{13} \mathrm{C}$ chemical shift values predicted for the old nimbosodione assignment by DFT (circles) and NN (triangles) methods versus experimental shift values. Both methods of chemical shift calculation show significant scattering of the calculated chemical shifts and significant deflection from the target line.

\section{Experimental.}

All calculations were performed on a PC computer (OS Windows 2000, SP4 ) with a 2,8 GHz processor and 2 Gbytes of memory.

\section{Conclusions.}

The elucidation of chemical structures from spectral data is one of the primary applications of molecular spectroscopy. Nowadays a combination of 1D and 2D NMR data are used as the primary data for this purpose. The two most important stages in solving a structure elucidation problem are the creation of structural hypotheses from the spectral data and verification of the hypotheses by chemical shift prediction. When conventional methods are used the structural hypotheses are derived by the researcher. Due to the biases of the investigators different 
researchers can suggest different structures. One consequence of this is that articles very frequently appear where previously suggested structures have been revised.

The selection of the right structure is usually attained by using chemical shift prediction for all suggested structures and the best structure is determined by structure ranking in an ascending order of deviation between the experimental and predicted NMR spectra. Until recently, empirical methods of NMR spectrum prediction were used, namely fragment-based, incremental and neural net algorithms ${ }^{[1]}$. During the last decade it was shown that the GIAO option of a quantum-mechanical DFT approximation could also be successfully used for this purpose.

This article considered a series of examples where several possible structures were derived from the NMR data by the investigators. When 2D NMR data were available we used the expert system Structure Elucidator ${ }^{[16,17]}$ for the generation of structural hypotheses, and the best structures were distinguished by empirical methods of chemical shift prediction implemented into the system. Results were compared with structures suggested by one or more groups of researchers and with the results of the most probable structure selection obtained when the QM methods were employed.

This work has shown that a systematic approach based on CASE principles allowed us to determine all structures which do not contradict the experimental data: the 1D and 2D NMR spectra and the molecular formula. Genuine structures are distinguished using a procedure of ranking structures in an ascending order of deviation between the experimental and calculated NMR spectra. Erroneous structures suggested by other researchers are also generated and are ranked lower in the ordered output file if the structures do not contradict the initial set of constraints. The program automatically rejects structures suggested by researchers that cannot be considered as consistent with the initial data. We believe that our observation that average chemical shift deviations found by our neural net approach (or incremental one) are frequently less than ones found by QM approach can be explained in the following way: the neural nets are trained using experimental NMR spectra which were recorded from molecules that likely exist in 
the most probable spatial configurations or in "average conformations". As a result the chemical shifts calculated by both methods are close for rigid structures and can also be close for flexible molecules.

When QM methods are used for structure elucidation it is desirable to reduce the set of structures as much as possible to prevent superfluous human labor and computational expenses. Minimization of a set of candidate structures and justification of structural hypotheses is achieved by application of Structure Elucidator. We conclude that the optimal way to solve the spectrum-structural problem is the creation of structural hypotheses using a 2D NMR based expert system and revealing the most probable structure(s) by empirical methods of chemical shift prediction. For additional verification of the preferred structures the application of QM prediction methods is very useful. Moreover, non-empirical methods can play a decisive role if the verified structures contain fragments which are absent from the database utilized for training the empirical methods of prediction. If 2D NMR data are not available then the application of fast but accurate incremental and neural net based algorithms ${ }^{[13,15]}$ for preliminary probability estimation of each structure suggested by a researcher can be extremely helpful. We suggest that a systematic approach based on an expert system application for structure elucidation from NMR data is the most practical and rational way for approaching the problem. We believe that the most convincing evidence of the effectiveness of the QM method as a tool for molecular structure elucidation could be examined if these methods were compared to the 2-3 top structures of output files produced by an expert system such as Structure Elucidator. 


\section{References}

[1] Elyashberg ME, Williams AJ, Martin GE. Prog. NMR Spectrosc. 2008; 53: 1-104.

[2] Nicolaou KC, Snyder SA. Angew. Chem. Int. Ed. 2005; 44: 1012-1044.

[3] Blinov KA, Elyashberg ME, Molodtsov SG, Williams AJ, Martirosian ER. Fresenius J. Anal. Chem. 2001; 369: 709-714.

[4] Lindel T, Junker J, Kock M. J. Mol. Model. 1997; 3: 364-368.

[5] Nuzillard J-M. Chin. J. Chem. 2003; 21: 1263-1267.

[6] Schulz KP, Korytko A, Munk ME. J. Chem. Inf. Comput. Sci. 2003; 43: 1447-1456.

[7] Elyashberg ME, Gribov LA, Serov VV. . Molecular spectral analysis and computer (in russian). Nauka: Moscow, 1980.

[8] Advanced Chemistry Development. ACD/NMR Predictors. Prediction suite includes ${ }^{1} \mathrm{H}$ ${ }^{13} \mathrm{C},{ }^{15} \mathrm{~N},{ }^{19} \mathrm{~F},{ }^{31} \mathrm{P}$ NMR prediction. http://www.acdlabs.com.

[9] Meiler J, Meusinger R, Will M. J. Chem. Inf. Comput. Sci. 2000; 40: 1169-1176.

[10] Pretsch E, Furst A, Badertscher M, Schaller RB, Munk ME. J. Chem. Inf. Comput. Sci. 1992; 32: 291-295.

[11] Robien W. Nachr. Chem. Tech. Lab. 1998; 46: 74-77.

[12] Schaller RB, Munk ME, Pretsch E. J. Chem. Inf. Model. 1996; 36: 239-243.

[13] Blinov KA, Smurnyy YD, Churanova TS, Elyashberg ME, Williams AJ. Chemom. Intell. Lab. Syst. (submitted for publication).

[14] Blinov KA, Smurnyy YD, Elyashberg ME, Churanova TS, Kvasha M, Steinbeck C, Lefebvre BE, J Williams A. J. Chem. Inf. Model. 2008; 48: 550-555.

[15] Smurnyy YD, Blinov KA, Churanova TS, Elyashberg ME, Williams AJ. J. Chem. Inf. Model. 2008; 48: 128-134.

[16] Blinov KA, Carlson D, Elyashberg ME, Martin GE, Martirosian ER, Molodtsov SG, Williams AJ. J. Magn Reson. Chem. 2003; 41: 359-372. 
[17] Elyashberg ME, Blinov KA, Molodtsov SG, Williams AJ, Martin GE. J. Chem. Inf. Comput. Sci. 2004; 44: 771-792.

[18] Elyashberg ME, Blinov KA, Williams AJ, Molodtsov SG, Martin GE. J. Chem. Inf. Model. 2006; 46: 1643-1656.

[19] Elyashberg ME, Blinov KA, Molodtsov SG, Smurnyy YD, Williams AJ, Churanova TS. Chemom. Intell. Lab. Syst. (submitted for publication).

[20] Bagno A, Rastrelli F, Saielli G. Chem. Eur. J. 2006; 12: 5514-5525.

[21] Bagno A, Saielli G. Theor. Chem. Acc. 2007; 117: 603-619.

[22] Balandina A, Saifina D, Mamedov V, Latypov S. J. Mol. Struc. 2006; 791: 77-81.

[23] Barone G, Gomez-Paloma L, Duca D, Silvestri A, Riccio R, Bifulco G. Chem. Eur. J. 2002; 8: 3233-3239.

[24] Barone V, Cimino P, Crescenzi O, Pavone M. J. Mol. Struc. 2007; 811: 323-335.

[25] Cimino P, Gomez-Paloma L, Duca D, Riccio R, Bifulco G. Magn. Reson. Chem. 2004; 42: S26-S33.

[26] Rychnovsky SD. Org. Lett. 2006; 8: 2895-2898.

[27] Schlegel B, Hartl A, Dahse H-M, Gollmick FA, Gräfe U, Dörfelt H, Kappes B. J. Antibiot. 2002; 55: 814-817.

[28] Porco JAJ, Su S, Lei X, Bardhan S, Rychnovsky SD. Angew. Chem. Int. Ed. 2006; 45: 14.

[29] Williams AJ, Elyashberg ME, Blinov KA, Lankin DC, Martin GE, Reynolds WF, Porco JA, Singleton CA, Su S. J. Nat. Prod. 2008; 71: 581-588.

[30] Sanz D, Claramunt RM, Saini A, Kumar V, Aggarwal R, Singh SP, Alkorta I, Elguero J. Magn. Reson. Chem. 2007; 45: 513-517.

[31] Balandina A, Mamedov V, Xavier F, Bruno F, Latypov S. Tetrahedron Lett. 2004; 45: 4003-4007. 
[32] Balandina AA, Mamedov VA, Khafizova EA, Latypov SK. Russ. Chem. Bull. 2006; 55: 2256-2264.

[33] Cambridge Soft Corporation. CambridgeSoft.com, 1985-2000.

[34] Modgraph. http://www.modgraph.co.uk/product_nmr.htm

[35] Robien W. http://felix.orc.univie.ac.at/ wr/csearch_server_info.html.

[36] Upstream Solutions GMBH. NMR Prediction Products (SpecTool):

http://www.upstream.ch/products/nmr.html

[37] Kim W-G, Kim J-W, Ryoo I-J, Kim J-P, Kim Y-H, Yoo I-D. Org. Lett. 2004; 6: 823-826.

[38] Steglich W, Hellwig V. Org. Lett., 2004; 6: 3175-3177.

[39] Molodtsov S.G., Elyashberg M.E., Blinov K.A., Williams A.J., Martin G.M., Lefebvre B. J. Chem. Inf. Comput. Sci. 2004; 44: 1737-1751.

[40] Elyashberg M.E., Blinov KA, Williams AJ, Molodtsov SG, Martin GE. J. Chem. Inf. Model. 2007; 47: 1053-1066.

[41] Martin GE, Zektzer AS. Two-Dimensional NMR Methods for Establishing Molecular Connectivity. VCH, New York,1988.

[42] Blümich B. Essential nmr for scientists and engineers. Springer: Berlin, 2005.

[43] Friebolin H. Basic one- and two-dimensional spectroscopy. WILEY-VCH: Weinheim, 2005.

[44] Ara I, Siddiqui BS, Faizi S, Siddiqui S. J. Nat. Prod. 1990; 53: 816-820.

[45] Li AP, Bie PY, Peng XS, Wu TX, Pan XF, Chan ASC, Yang TK. Synth. Commun. 2002; 32: 605-610. 


\section{TABLES}

Table 1. Experimental and calculated chemical shifts in ppm related to the four carbon atoms of isomers 24 and 25.

\begin{tabular}{|c|c|c|c|c|c|}
\hline Exp1 & Exp2 & $\begin{array}{c}\text { DFT } \\
\mathbf{2 4}\end{array}$ & $\begin{array}{c}\text { NN } \\
\mathbf{2 4}\end{array}$ & $\begin{array}{c}\text { DFT } \\
\mathbf{2 5}\end{array}$ & $\begin{array}{c}\text { NN } \\
\mathbf{2 5}\end{array}$ \\
\hline 147.56 & 147.12 & 141.69 & 147.13 & 141.94 & 148.14 \\
\hline 156.97 & 157.65 & 149.8 & 155.22 & 149.38 & 154.56 \\
\hline 143.78 & 144.87 & 136.75 & 145.01 & 135.92 & 143.29 \\
\hline 141.33 & 140.12 & 132.29 & 138.8 & 133.17 & 143.3 \\
\hline
\end{tabular}

Table 2. Results of comparison of four experimental chemical shifts taken from each of the two spectra with corresponding calculated chemical shifts.

\begin{tabular}{|c|c|c|c|c|}
\hline Spectra & DFT & NN & DFT & NN \\
& $\mathbf{2 4}$ & $\mathbf{2 4}$ & $\mathbf{2 5}$ & $\mathbf{2 5}$ \\
\hline 1 & $\mathrm{R}^{2}=0.9746$ & $\mathrm{R}^{2}=0.9428$ & $\mathbf{R}^{2}=\mathbf{0 . 9 7 5 5}$ & $\mathbf{R}^{2}=\mathbf{0 . 9 7 1 1}$ \\
& $\mathrm{SE}=1.46$ & $\mathrm{SE}=1.99$ & $\mathbf{S E}=\mathbf{1 . 3 8}$ & $\mathbf{S E}=\mathbf{1 . 1 1}$ \\
\hline 2 & $\mathbf{R}^{\mathbf{2}}=\mathbf{0 . 9 7 1 9}$ & $\mathbf{R}^{\mathbf{2}}=\mathbf{0 . 9 7 8 5}$ & $\mathrm{R}^{2}=0.9497$ & $\mathrm{R}^{2}=0.9149$ \\
& $\mathbf{S E}=\mathbf{1 . 5 4}$ & $\mathbf{S E}=\mathbf{1 . 2 2}$ & $\mathrm{SE}=1.97$ & $\mathrm{SE}=1.91$ \\
\hline
\end{tabular}

Table 3. Statistical parameters calculated for structures 26-28.

\begin{tabular}{|l|l|l|l|}
\hline & \multicolumn{1}{|c|}{$\mathbf{2 6}$} & \multicolumn{1}{|c|}{$\mathbf{2 7}$} & \multicolumn{1}{|c|}{$\mathbf{2 8}$} \\
\hline$R^{2}(\mathrm{QM})$ & 0.9975 & 0.9925 & 0.9844 \\
\hline$R^{2}(\mathrm{NN})$ & 0.9975 & 0.9931 & 0.9944 \\
\hline$d_{Q}, \mathrm{ppm}$ & 1.82 & 3.51 & 4.99 \\
\hline$d_{N}, \mathrm{ppm}$ & 2.06 & 3.32 & 3.36 \\
\hline
\end{tabular}

